\title{
QUASISYMMETRIC AND LIPSCHITZ APPROXIMATION OF EMBEDDINGS
}

\author{
J. LUUKKAINEN and P. TUKIA
}

\section{Introduction}

This paper is concerned with the following concepts. Let $(X, d)$ and $\left(Y, d^{\prime}\right)$ be metric spaces and let $f: X \rightarrow Y$ be an embedding. Then $f$ is said to be bilipscinitz (or L-bilipschitz) if, for some $L \geqq 1$,

$$
d(x, y) / L \leqq d^{\prime}(f(x), f(y)) \leqq L d(x, y)
$$

for all $x, y \in X$. It is called quasisymmetric (or $\eta$-quasisymmetric) if, for some homeomorphism $\eta: R_{+}^{1} \rightarrow R_{+}^{1}$,

$$
d^{\prime}(f(a), f(x)) \leqq \eta(t) d^{\prime}(f(b), f(x))
$$

whenever $a, b, x \in X, t \geqq 0$, and $d(a, x) \leqq t d(b, x)$. The above condition is motivated by the properties of quasiconformal maps: For instance, a homeomorphism of $R^{n}$ is quasiconformal if and only if it is quasisymmetric. Like bilipschitz embeddings, quasisymmetric embeddings form a category: The composite of two quasisymmetric embeddings is quasisymmetric, and so is the inverse of a quasisymmetric homeomorphism (cf. [38]). Every bilipschitz embedding is quasisymmetric.

A map $f: X \rightarrow Y$ is called an LQS immersion if every point of $X$ has a neighborhood on which $f$ is quasisymmetric. It is called a LIP immersion if every point of $X$ has a neighborhood on which $f$ is bilipschitz. We let CAT denote either LQS or LIP. A CAT embedding or a CAT homeomorphism is a CAT immersion which is an embedding or a homeomorphism, respectively. It is obvious that the inverse of a CAT homeomorphism is a CAT homeomorphism. Every LQS embedding of a compact space is quasisymmetric by [38, Theorem 2.23], and every LIP embedding of a compact space is bilipschitz. A piecewise linear (PL) embedding between polyhedra in Euclidean spaces is a CAT embedding. We call a separable metric space a metric CAT $n$-manifold, $n \geqq 0$, if every point has a closed neighborhood CAT homeomorphic to the cube $[-1,1]^{n}$ in $R^{n}$.

There is a familiar and apparently more general alternative way to define CAT manifolds and CAT immersions based on atlases; see 1.3. As LQS atlases are the 
same as locally quasiconformal atlases, LQS manifolds are also called quasiconformal manifolds. The two definitions of LIP or Lipschitz manifolds were proved to be (essentially) equivalent in [26, Theorems 3.5 and 4.2]. J. Väisälä raised the question whether this also holds for LQS manifolds. Our main result implies that such is really the case.

Section 1 is preliminary except for Theorem 1.14, where it is proved that every LIP embedding between LIP manifolds is locally flat if the codimension is at least three. Lemma 1.9 recapitulates known results about PL approximation of embeddings from dimension $n$ into dimension $q$. It is valid if either $q \geqq n=1$ or $n \geqq 2, q \geqq n+3$ or $(n, q) \in\{(2,2),(2,3),(3,3)\}$. We call these pairs $(n, q)$ admissible.

In Section 2 we prove that if $(n, q)$ is admissible, $Y \subset X \subset R^{n}$, and $Y$ is open in $R^{n}$, then every quasisymmetric embedding $f: X \rightarrow R^{q}$ can be approximated by quasisymmetric embeddings which coincide with $f$ on $X \backslash Y$ and are PL on $Y$. In fact, we consider here more general, if quite special, polyhedra $Y$; these are open in $X$ and have a certain decomposition into $n$-cubes. In the case $n=q \leqq 3$ similar but simpler problems for bilipschitz or quasiconformal embeddings are studied in [41, Theorem 2.4] and [18, Theorems 2.1 and 3.1]. Our proof is similar to the ones in [41] and [18]. It is based on Lemma 1.9 and the finiteness idea of Carleson in [10], which can be used by virtue of a compactness property of quasisymmetric embeddings.

In Section 3 we apply results of Section 2 and obtain analogous results for bilipschitz emteddings.

Using these theorems we prove in Section 4 the main result of this paper, Theorem 4.4. In a simplified form it states (cf. 4.14.4) that if $M$ and $N$ are CAT manifolds of dimensions $n$ and $q$, respectively, such that $(n, q)$ is admissible, then every embedding $f: M \rightarrow N$ can be approximated by CAT embeddings in the source majorant topology. A special case of Theorem 4.4 for CAT $=$ LIP and $n=q \leqq 3$ is given in [37, Theorem 2], and our proof is a modification of the proof in [37]. As a corollary we get the result that $M$ can be CAT embedded into $R^{2 n+1}(n \geqq 0)$, from which it follows that the two definitions of CAT manifolds are equivalent. Further, by recent results about topological embeddings, $R^{2 n+1}$ can here be replaced by $R^{2 n}$ ( $\left.n \geqq 1\right)$ and, if the target majorant topology is used and $q \geqq 2 n+1$, it suffices to assume that $f$ is only a continuous map.

Acknowledgements. The first-named author wishes to thank the Government of France for a scholarship and the University of Paris XI at Orsay for the hospitality he enjoyed during the completion of this paper. We are grateful to J. Dancis, L. Siebenmann, and J. Väisälä for $1.14,1.16$, and 1.17 . 


\section{Terminology and preliminary results}

1.1. Notation. The letters $n, q$ denote non-negative integers. Let $R^{n}$ be the Euclidean $n$-space, $R_{+}^{n}=\left\{x \in R^{n} \mid x_{n} \geqq 0\right\}, \quad R^{n-1} \equiv \partial R_{+}^{n}, \quad I^{n}=[-1,1]^{n}, \quad J^{n}=(-1,1)^{n}$, $J_{+}^{n}=J^{n} \cap R_{+}^{n}, I^{n}(r)=r I^{n}$ and $J^{n}(r)=r J^{n}$ for $r>0$, and $I=[0,1]$. Let $e_{1}=$ $(1,0, \ldots, 0) \in R^{n}$. If $S$ is a topological space, let $C_{+}(S)=\{f \mid f: S \rightarrow(0, \infty)$ continuous\}. If $S$ is a set and $f, g: S \rightarrow R^{n}$, we write $d(f, g)=\sup \{\mid f(x)-g(x) \| x \in S\}$.

1.2. Weakly quasisymmetric embeddings. An embedding $f$ of a metric space $(X, d)$ into a metric space $\left(Y, d^{\prime}\right)$ is called weakly quasisymmetric if there is $H \geqq 1$ such that $d^{\prime}(f(a), f(x)) \leqq H d^{\prime}(f(b), f(x))$ whenever $a, b, x \in X$ and $d(a, x) \leqq d(b, x)$; then $f$ is also said to be weakly H-quasisymmetric. This concept, too, was considered in [38]. Every quasisymmetric embedding is weakly quasisymmetric. Every weakly quasisymmetric embedding $f: S \rightarrow R^{q}, S \subset R^{n}$, has a unique extension to a closed embedding $\bar{f}: \bar{S} \rightarrow R^{q}$. If $f$ is $L$-bilipschitz, this is well-known, and $\bar{f}$ is $L$-bilipschitz. If $f$ is $\eta$-quasisymmetric, this follows from [38, Theorems 2.24 and 2.25], and $\bar{f}$ is, $\eta$-quasisymmetric. The general case follows from [33, Lemmas 2, 4, and 5] (the case $n=q=2$ considered in [33] can be generalized in the obvious way). Moreover, in [33] one does not assume that $f$ is an embedding but only that $f$ is injective. (For a similar definition for quasisymmetric embeddings, see [38, Theorem 2.21].) We do not know whether $\bar{f}$ is always weakly quasisymmetric. (However, it is easy to see that if $S$ is open and convex and if $f$ is weakly $H$-quasisymmetric, then $\bar{f}$ is weakly $H$-quasisymmetric.)

1.3. Atlases. We give the definition of CAT manifolds in the atlas sense. Let CAT $(n)$ be the category whose objects are open subsets of $R^{n}$ and of $R_{+}^{n}$ and whose morphisms are CAT homeomorphisms. Then CAT $(n)$ is a pseudogroup of transformations in a slightly more general sense than in $[20$, p. 1]. Consider a homeomorphism $f: U \rightarrow V$, where $U, V$ are open either in $R^{n}$ or in $R_{+}^{n}$. By [38, Theorem 2.16], [43, Theorems 2.3 and 2.4], and [40, Theorem 35.2], the following conditions are equivalent for a point $x \in U$ : (1) $f$ is quasisymmetric on a neighborhood of $x$ in $U$; (2) $f$ is weakly quasisymmetric on a neighborhood of $x$ in $U$; (3) (for $n \geqq 2$ ) there is an open neighborhood $W$ of $x$ in $R^{n}$ such that $f \mid W \cap \operatorname{int} U$ is a quasiconformal embedding; (4) (for $n \geqq 2$ ) there is an open neighborhood $W$ of $x$ in $R^{n}$ such that $f \mid W \cap U$ extends to a quasiconformal embedding of $W$ into $R^{n}$.

A CAT $(n)$ atlas $\mathscr{A}$ on a topological space $M$ is a family of pairs $(U, h)$, called charts, such that the $U$ 's are open sets of $M$ covering $M, h$ is a homeomorphism of $U$ onto an open subset of $R^{n}$ or of $R_{+}^{n}$, and for charts $(U, h),\left(U^{\prime}, h^{\prime}\right)$, the homeomorphism $h^{\prime} h^{-1}: h\left(U \cap U^{\prime}\right) \rightarrow h^{\prime}\left(U \cap U^{\prime}\right)$ belongs to CAT $(n)$. If $M$ is a separable metrizable space and if $\mathscr{A}$ is a CAT $(n)$ atlas on $M$ which is maximal with respect to inclusion, we call the pair $(M, \mathscr{A})$ a CAT $n$-manifold and $\mathscr{A}$ a CAT structure on $M$. The terms quasiconformal structure and Lipschitz structure are also used. 
(We could also define a CAT structure as an equivalence class of CAT (n) atlases, two atlases being equivalent if their union is a $\operatorname{CAT}(n)$ atlas.)

Let $(M, \mathscr{A})$ be a CAT manifold. The underlying space $M$ is a topological manifold, whose interior int $M$ and boundary $\partial M$ inherit a CAT structure from $\mathscr{A}$ in a natural way. If $A$ and $B$ are subsets of CAT manifolds and if $X$ is a metric space, we can define CAT immersions $A \rightarrow B, A \rightarrow X$, and $X \rightarrow A$ in a familiar way using charts (cf. $[42,1.8]$ ). One defines similarly CAT embeddings and CAT homeomorphisms. If $(N, \mathscr{B})$ is a CAT manifold such that $N \subset M$ and that the inclusion of $(N, \mathscr{B})$ into $(M, \mathscr{A})$ is a CAT embeding, we call $(N, \mathscr{B})$ a CAT submanifold of $M$. Suppose that $\partial M=\emptyset$. Then a CAT $q$-submanifold $N$ of $M$ is said to te locally CAT flat if for each $x \in N$ there is $(U, h) \in \mathscr{A}$ such that $x \in U$ and $h(U \cap N)$ equals $h U \cap R^{q}$ or $h U \cap R_{+}^{q}$. A CAT embedding is called locally CAT flat if its image is locally CAT fiat.

Every metric CAT $n$-manifold $M$ (in the sense of the Introduction) has a natural CAT structure consisting of all pairs $(U, h)$ where $U$ is open in $M$ and $h$ is a CAT homeomorphism of $U$ onto an open set in $R^{n}$ or in $R_{+}^{n}$. Moreover, if $A \subset M$, the two definitions of CAT immersions of $A$ or into $A$ coincide. We consistently define a subset $N$ of $M$ to be a CAT submanifold of $M$ if it is a metric CAT manifold in the induced metric.

In 4.7 we will see that every CAT manifold has a metric which induces the original CAT structure.

In 4.11 we will need the fact that every CAT $n$-manifold can be CAT embedded into a CAT $n$-manifold without boundary. For this reason we construct the double of a CAT manifold.

1.4. Lemma. Let $(M, \mathscr{A})$ be a CAT n-manifold. Then there exists a CAT n-manifold $(D M, \mathscr{B})$, called the double of $M$, with the following properties: $D M$ contains CAT submanifolds $M_{1}, M_{2}$ such that $D M=M_{1} \cup M_{2}, M_{1} \cap M_{2}=\partial M_{1}=$ $\partial M_{2}$, and there are CAT homeomorphisms $f_{i}: M \rightarrow M_{i}$ such that $f_{1}\left|\partial M=f_{2}\right| \partial M$. The triple $\left(D M, f_{1}, f_{2}\right)$ is unique up to a CAT homeomorphism except possibly when $\mathrm{CAT}=\mathrm{LQS}, n=1$, and $\partial M \neq \emptyset$, in which case, however, $\left(D M, M_{1}, M_{2}\right)$ is unique up to a CAT homeomorphism. Moreover, $\partial D M=\emptyset$, and the submanifolds $M_{1}, M_{2}, M_{1} \cap M_{2}$ are locally CAT flat in $D M$.

Proof. We only consider $\mathrm{CAT}=\mathrm{LQS}$, tecause for $\mathrm{CAT}=\mathrm{LIP}$ one can give a similar but slightly simpler proof and because one has already proved this case by another method in [26, Theorem 3.13]. It is well-known that the lemma holds for topological manifolds and homeomorphisms (i.e., if CAT is replaced by TOP). This gives us the manifold $D M$ and the homeomorphisms $f_{i}$. We construct an LQS structure on $D M$ as follows. Define $p: R^{n} \rightarrow R^{n}$ by $p(x)=\left(x_{1}, \ldots, x_{n-1},-x_{n}\right)$. For each chart $(U, h) \in \mathscr{A}$ with $U \cap \partial M=\emptyset$ we have the charts $\left(f_{i} U, h f_{i}^{-1} \mid f_{i} U\right)$, $i=1,2$, of $D M$. For each chart $(U, h) \in \mathscr{A}$ with $U \cap \partial M \neq \emptyset$ we define a chart $\left(U^{*}, h^{*}\right)$ of $D M$ as follows. Since $h U$ is open in $R_{+}^{n}$, the set $V=h U \cup p h U$ is open 
in $R^{n}$. Let $U^{*}=f_{1} U \cup f_{2} U$; then $U^{*}$ is open in $D M$. We define a homeomorphism $h^{*}: U^{*} \rightarrow V$ by $h^{*}(x)=h f_{1}^{-1}(x)$ if $x \in f_{1} U$ and $h^{*}(x)=p h f_{2}^{-1}(x)$ if $x \in f_{2} U$. These charts form an atlas $\mathscr{B}_{0}$ on $D M$. One can use [40, Theorem 35.2] for $n \geqq 2$ and [24, II, Lemma 7.1 and (7.2)] for $n=1$ to see that $\mathscr{B}_{0}$ is an LQS (n) atlas. The LQS structure $\mathscr{B}$ determined by $\mathscr{B}_{0}$ depends only on $\mathscr{A}$. The sets $M_{1}, M_{2}$, and $M_{1} \cap M_{2}$ are locally LQS flat LQS submanifolds of $(D M, \mathscr{B})$, and the homeomorphisms $f_{i}$ are LQS.

To show the uniqueness, let $\left(D^{\prime} M, M_{1}^{\prime}, M_{2}^{\prime}, f_{1}^{\prime}, f_{2}^{\prime}\right)$ have the properties of $\left(D M, M_{1}, M_{2}, f_{1}, f_{2}\right)$ listed in the first part of the lemma. Let $g: D M \rightarrow D^{\prime} M$ be the unique homeomorphism with $g f_{i}=f_{i}^{\prime}, i=1,2$. Since $M_{1} \cap M_{2}$ is locally LQS flat in $D M$, it follows from [40, Theorem 35.1] that $g$ is LQS if $n \geqq 2$. If $n=1$ and $\partial M=\emptyset$, then trivially $g$ is LQS. If $n=1$ and $\partial M \neq \emptyset$, the classification of LQS 1-manifolds in 4.8 implies that there exists an LQS homeomorphism $h: D M \rightarrow D^{\prime} M$ with $h M_{i}=M_{i}^{\prime}$. The existence of an LQS homeomorphism of $\left(D M, M_{1}, M_{2}\right)$ onto $\left(D^{\prime} M, M_{1}^{\prime}, M_{2}^{\prime}\right)$ implies that the submanifolds $M_{1}^{\prime}, M_{2}^{\prime}$, $M_{1}^{\prime} \cap M_{2}^{\prime}$ of $D^{\prime} M$ are locally LQS flat (this can also be proved directly).

1.5. Example. Define a homeomorphism $g: J^{1} \rightarrow J^{1}$ by $g(x)=x$ if $x \leqq 0$ and $g(x)=x^{2}$ if $x \geqq 0$. Then $g \mid(-1,0]$ and $g[[0,1)$ are quasisymmetric, but $g$ is not LQS. This implies that in 1.4 the uniqueness of $\left(D M, f_{1}, f_{2}\right)$ does not hold for $n=1$.

1.6. Function spaces. Let $C(X, Y)$ denote the set of all continuous maps of a metrizable space $X$ into a metrizable space $Y$. Let $f \in C(X, Y)$. We call $f$ proper if the inverse image of every compact set is compact. An embedding of $X$ into $Y$ is closed if and only if it is proper. Let $d$ be a metric for $Y$. The sets

$$
U_{d}(f, \varepsilon)=\{g \in C(X, Y) \mid \forall x \in X, d(f(x), g(x))<\varepsilon(x)\}
$$

for $\varepsilon \in C_{+}(X)$ form a neighborhood basis of $f$ in the source majorant topology of $C(X, Y)$, which is independent of $d$. If $X$ and $Y$ are locally compact and $f$ is proper, there is a neighborhood $U_{d}(f, \varepsilon)$ whose elements are proper. The sets

$$
N(f, \mathscr{U})=\{g \in C(X, Y) \mid \forall x \in X \exists U \in \mathscr{U}, f(x), g(x) \in U\},
$$

where $\mathscr{U}$ is an open cover of $Y$, form a neighborhood basis of $f$ in the target majorant topology of $C(X, Y)$. For every $N(f, \mathscr{U})$ there is $U_{d}(f, \varepsilon) \subset N(f, \mathscr{U})$. Conversely, if $f$ is proper, every $U_{d}(f, \varepsilon)$ contains an $N(f, \mathscr{U})$. It is easy to prove that if $X, Y$, and $Z$ are metrizable spaces, then the map $C(X, Y) \times C(Y, Z) \rightarrow C(X, Z),(f, g) \rightarrow g f$, is continuous whenever each function space has the target majorant topology.

These facts are well-known; some references are given in [25, 1.2].

In the next three lemmas we have collected known PL approximation results.

1.7. Lemma. Let $(n, q)$ be admissible, $M$ a PL $n$-manifold, $N$ a PL $q$-manifold with $\partial N=\varnothing, f: M \rightarrow N$ an embedding, $d$ a metric for $N$, and $\varepsilon \in C_{+}(M)$. Then there is a PL embedding $g: M \rightarrow N$ in $U_{d}(f, \varepsilon)$. 
Proof. The set $f M$ is locally compact and thus closed in an open set $N_{0}$ of $N$; hence replacing $N$ by $N_{0}$ we may assume that $f$ is closed. One can give an elementary proof in the case $q \geqq n=1$. The case $n \geqq 2, q \geqq n+3$ follows both from [28, Theorem 3] and [6, Theorem 1] (or [13, Theorem 8.1]). For the case $n=q=2$, see [30, Theorem 6.4]. The case $n=q=3$ is proved in [4, Theorem 9]. Consider finally the case $(n, q)=(2,3)$. By each of [3, Theorem 7], [4, Theorem 5], and [4, Theorem 10], there is an embedding $h: f M \rightarrow N$ such that $h f \in U_{d}(f, \varepsilon / 2)$ and such that $M^{\prime}=h f M$ is a subpolyhedron and thus, by [30, Theorem 4.9], a PL submanifold of $N$. Hence there is a PL homeomorphism $g_{0}: M \rightarrow M^{\prime}$ in $U_{d}(h f, \varepsilon / 2)$; cf. [30, Theorem 6.4] or [5, Theorem 4.6]. Then the PL embedding $g: M \rightarrow N$ defined by $g_{0}$ is in $U_{d}(f, \varepsilon)$.

1.8. Lemma. Let $n, q, M, N$, and $d$ be as in 1.7 with $n \geqq 2$, let $f: M \rightarrow N$ be a closed embedding, and $\varepsilon \in C_{+}(N)$. Then there is $\delta \in C_{+}(M)$ with the following property: If $g_{i}: M \rightarrow N, i=0,1$, is a PL embedding in $U_{d}(f, \delta)$, there is a $\mathrm{PL}$ homeomorphism $h: N \rightarrow N$ in $U_{d}\left(\mathrm{id}_{N}, \varepsilon\right)$ such that $h g_{0}=g_{1}$.

Proof. The case $n \geqq 2, q \geqq n+3$ follows from each of [7, Theorem 1], [28, Theorem 2], and [13, Corollary 6.1], the case $2 \leqq n \leqq q=3$ from [11, Theorem 7.1], and the case $n=q=2$ from [11, Theorem 7.2]. (These results give, moreover, a PL ambient $\varepsilon$-isotopy from id ${ }_{N}$ to $h$.)

1.9. Lemma. Let $n, q, M, N, f, d$, and $\varepsilon$ be as in 1.7. Then for each PL $n$-submanifold $M_{1}$ of $M$ which is closed in $M$, there exists $\delta \in C_{+}\left(M_{1}\right)$ with the following property: If $g: M_{1} \rightarrow N$ is a PL embedding in $U_{d}\left(f \mid M_{1}, \delta\right)$, there is a PL embedding $g^{*}: M \rightarrow N$ in $U_{d}(f, \varepsilon)$ which extends $g$.

Proof. If $M_{1}=\emptyset$, the lemma reduces to 1.7. We may assume that $f$ is closed; cf. the proof of 1.7. An elementary proof can be given if $q \geqq n=1$. Suppose that $n \geqq 2$. By 1.6 there are $\delta_{0} \in C_{+}(M)$ and $\varepsilon_{0} \in C_{+}(N)$ such that if $f_{0} \in U_{d}\left(f, \delta_{0}\right)$ and $h_{0} \in U_{d}\left(\mathrm{id}_{N}, \varepsilon_{0}\right)$, then $h_{0} f_{0} \in U_{d}(f, \varepsilon)$. Let $\delta \in C_{+}\left(M_{1}\right)$ be the function which 1.8 gives if we substitute $M \mapsto M_{1}, f \mapsto f \mid M_{1}, \varepsilon \mapsto \varepsilon_{0}$. Now let $g \in U_{d}\left(f \mid M_{1}, \delta\right)$ be a PL embedding. Choose $\delta_{1} \in C_{+}(M)$ with $\delta_{1} \mid M_{1}=\delta$ and set $\delta^{\prime}(x)=\min \left(\delta_{0}(x), \delta_{1}(x)\right)$ for $x \in M$. By 1.7 we can choose a PL embedding $g^{\prime} \in U_{d}\left(f, \delta^{\prime}\right)$. Then there is a PL homeomorphism $h \in U_{d}\left(\mathrm{id}_{N}, \varepsilon_{0}\right)$ with $h\left(g^{\prime} \mid M_{1}\right)=g$. Hence $g^{*}=h g^{\prime}: M \rightarrow N$ is a PL embedding in $U_{d}(f, \varepsilon)$ with $g^{*} \mid M_{1}=g$.

1.10. Remarks. 1. For $n \geqq 1, q \geqq 2 n+1$, Lemma 1.9 also follows from PL general position results ([16, Lemma 4.8, p. 102]; cf. [25, Lemma 3.5]). Moreover, the manifold pair $\left(M, M_{1}\right)$ can now be replaced by a pair $\left(X, X_{1}\right)$, where $X$ is a polyhedron with $\operatorname{dim} X \leqq n$ and $X_{1}$ is a closed subpolyhedron of $X$.

2. We will only need 1.9 in the case where $M_{2}=\overline{M \backslash M_{1}}$ is a PL $n$-manifold and $M_{1} \cap M_{2}$ a PL $(n-1)$-manifold. If $n=q=2$ or 3 , there is the following proof for this special case of 1.9. Suppose first that $M$ is compact. Then, if $n=3$, the 
proof is given in [29, Lemma 4] (cf. [2, Theorem 1']), and for $n=2$ the result certainly also holds with a simpler proof. The general case can easily be deduced from this special case; for example, one can proceed as in the proof of [2, Theorem 3].

3. For $n \geqq 2, q \geqq n+3$, Lemmas $1.7,1.8$, and 1.9 also hold if $M$ and $\left(M, M_{1}\right)$ are replaced, respectively, by a polyhedron $X$ or a polyhedral pair $\left(X, X_{1}\right)$ as in 1.10.1 above; this follows from the results quoted in the proofs. However, we do not know whether this holds for 1.9 if $2 \leqq n \leqq q \leqq 3$ (or if $n=1, q=2$ ).

4. Lemma 1.9 holds trivially if $q \geqq n=0$. For $n=q \geqq 5$, Lemma 1.7 fails by [19], and 1.9 is not true even if $N=R^{q}$ (cf. [41, 2.3]). By [27], 1.7 does not hold if $q=n+2 \geqq 4$, but if $n=2, q=4$, and $M=I^{2}$, it holds by [45, Theorem 1]. The case $q=n+1 \geqq 4$ seems to be unsettled.

1.11. We need the rest of this section only for applications in Section 4. We now give some definitions. A set $A$ in a metric space $X$ is called a $Z^{n}$-set in $X$ if every continuous map of $I^{n}$ into $X$ can be uniformly approximated by continuous maps into $X \backslash A$. An embedding of a space into $X$ is called a $Z^{n}$-embedding if its image is a $Z^{n}$-set in $X$. Let $(X, d)$ and $\left(Y, d^{\prime}\right)$ be metric spaces. A map $f: X \rightarrow Y$ is said to be LIP if for every point $p$ of $X$ there is a neighborhood $U$ of $p$ and $L \geqq 0$ such that $d^{\prime}(f(x), f(y)) \leqq L d(x, y)$ for all $x, y \in U$. If $A, B$ are subsets of LIP manifolds, the definitions of LIP maps $A \rightarrow B, A \rightarrow X$, and $X \rightarrow A$ are obvious; cf. 1.3.

1.12. Lemma. Let $n \geqq 0, q \geqq 2 n+1, X$ a separable metrizable space with $\operatorname{dim} X \leqq n, \quad N$ a topological q-manifold, $f: X \rightarrow N$ continuous, $C$ a locally compact closed subset of $X, f \mid C$ a closed $Z^{n}$-embedding, and $\mathscr{U}$ an open cover of $N$. Then $N(f, \mathscr{U})$ contains an embedding $g$ with $g|C=f| C$.

Proof. The case $C=\emptyset$ is proved in [25, Theorem 5.6]; if, in addition, $N$ can be embedded into $R^{q}$, a simpler proof is given in [25, Theorem 2.1]. The general case is due to Heisey and Toruńczyk; see [25, Theorem E of the Introduction].

1.13. Lemma. Let $X$ be a separable metric space, $N$ a metric LIP $q$-manifold, $f: X \rightarrow N$ LIP, and the q-dimensional Hausdorff measure $\mathscr{H}^{q}\left(X \times I^{n}\right)=0$, where $n<q$. Then $f X$ is a $Z^{n}$-set in $N$.

Proof. Let $g: I^{n} \rightarrow N$ be continuous and $U$ a uniform neighborhood of $g$. Using a topological collar of $\partial N$ in $N$ we may assume that $g I^{n} \subset$ int $N$ ([25, Lemma 2.3]). By [26, Corollary 5.18] we may further assume that $g$ is LIP. Then $\mathscr{H}^{q}\left(f X \times g I^{n}\right)=0$. Hence by a slight generalization of [26, Theorem 6.9] there is $h \in U$ with $h I^{n} \cap f X=\emptyset$.

The following theorem is related to Theorem 4.4. For $q \geqq 5$ it is an observation of L. Siebenmann. 
1.14. Theorem. Let $n \geqq 0, q \geqq n+3, M$ a LIP $n$-manifold, $N a$ LIP $q$-manifold, $\partial N=\emptyset$, and $f: M \rightarrow N$ an embedding which is a LIP map. Then fis locally flat.

Proof. We may assume that $M=I^{n}$ and $N=R^{q}$. The case $n=0$ is trivial. For $q \geqq 3 n+1$ the theorem follows from [44, Theorem 3.8]. This implies the case $n=1$. Let $n \geqq 2$. By [7, Theorem 2], 1.7, and [35, Theorem 1.7.2, p. 34], it suffices to prove that if $x \in f I^{n}, \varepsilon>0$, and $B=\left\{y \in R^{q}|| x-y \mid<\varepsilon\right\}$, then $U=B \backslash f I^{n}$ is simply connected. By [17, Corollary 1, p. 48], $U$ is connected. Let $g: \partial I^{2} \rightarrow U$ be continuous. There is a continuous extension $g_{1}: I^{2} \rightarrow B$ of $g$. Choose a LIP approximation $h: I^{2} \rightarrow B$ of $g_{1}$. By [26, Theorem 6.5], $\left(h I^{2}+y\right) \cap f I^{n}=\emptyset$ for almost all $y \in R^{q}$. If $d\left(g_{1}, h\right)$ and $|y|$ are small enough, $g^{\prime}=h+y$ is a map $I^{2} \rightarrow U$ such that $g$ and $g^{\prime} \mid \partial I^{2}$ are homotopic in $U$. (The existence of $g^{\prime}$ also follows from 1.13.) Hence $g$ is null-homotopic in $U$.

1.15. Lemma. Let $X$ be a locally compact separable metrizable space with $\operatorname{dim} X \leqq n \leqq 0$. Then there is a closed embedding of $X$ into $R^{2 n+1}$.

Proof. This follows easily from 1.12 . We can also reduce it to a classical special case of 1.12. Choose a compact metric space $Y$ containing $X$ as a subspace with $Y \backslash X=\{p\}$. Then $\operatorname{dim} Y=\operatorname{dim} X$ by [17, Corollary 2, p. 32]. Hence [17, Theorem V 2] gives an embedding $f: Y \rightarrow I^{2 n+1} \times\{1\}$. Then $f X$ is closed in $\partial I^{2 n+2} \backslash\{f(p)\} \approx$ $R^{2 n+1}$.

The following lemma is due to J. Väisälä.

1.16. Lemma. Let $n \geqq 4$, and let $X$ be a locally connected locally compact separable metrizable space with $\operatorname{dim} X \leqq n-2$ such that for each component $X_{j}$ of $X$ there is a closed embedding $f_{j}: X_{j} \rightarrow R^{n}$. Then there is a closed embedding $f: X \rightarrow R^{n}$.

In addition, if $X$ is a topological manifold and each $f_{j}$ is locally flat, $f$ can also be chosen to be locally flat.

Proof. We show first that if $X$ is connected, there is a closed embedding $g: X \rightarrow J^{n}$ with $\partial J^{n} \pitchfork \overline{g X}$. Choose a closed embedding $f: X \rightarrow R^{n}$. There is a closed PL embedding $\alpha: R_{+}^{1} \rightarrow R^{n}$ with $\alpha R_{+}^{1} \cap f X=\emptyset$. By [35, Theorem 3.4.3, p. 109], $\alpha$ is flat. Thus we get a closed embedding $h: X \rightarrow J^{n}$ with $h X \cap J_{+}^{1}=0$. Obviously, there is a homeomorphism $\varphi: J^{n} \rightarrow J^{n}$ with $e_{1} \notin \overline{\varphi h X}$. Then $g=\varphi h$ is the required map.

In the general case each component $X_{j}$ of $X$ is open and we may assume that $j \in\{1,2, \ldots\}$. Let $H^{n}=$ int $R_{+}^{n} \approx R^{n}$ and $U_{j}=J^{n} \cap H^{n}+3 j e_{1}$. The first part of the proof implies that there is a closed embedding $g_{j}: X_{j} \rightarrow U_{j}$ with $\overline{g_{j} X_{j}} \cap \partial U_{j} \subset \partial H^{n}$. Then $f=\cup_{j} g_{j}: X \rightarrow H^{n}$ is a closed embedding.

The assertion concerning local flatness can be proved similarly.

1.17. Lemma. Let $M$ be a topological $n$-manifold, $n \geqq 1$. Then there exists a closed locally flat embedding of $M$ into $R^{2 n}$. 
Proof. Replacing $M$ by its double, we may assume that $\partial M=\emptyset$. The case $n=1$ is trivial. Suppose $n=2$ or 3 . By the tubular neighborhood theorem it suffices to prove that $M$ is homeomorphic to a closed $C^{\infty}$-differentiable submanifold of $R^{2 n}$. By [30, Theorems 4.8, 8.3, 23.1, and 35.3], $M$ is homeomorphic to a PL manifold. Hence, by [8, Theorem III], $M$ is homeomorphic to a $C^{1}$-differentiable manifold $N$. Thus, by [31, Theorem 4.8], it suffices to show that if $N$ is connected, there exists a closed $C^{1}$-embedding $f: N \rightarrow R^{2 n}$ with $f N \subset J^{2 n-1} \times R^{1}$. If $N$ is compact, this follows from [46, Theorem 5]. If $N$ is non-compact, there is a $C^{1}$-embedding $f_{0}: N \rightarrow J^{2 n-1}$ by [15, Theorem 4.6]. Choose a proper $C^{1}$-differentiable function $f_{1}: N \rightarrow R^{1}$; then $f=\left(f_{0}, f_{1}\right)$ is the required map.

Suppose now $n \geqq 4$. By 1.16 we may assume that $M$ is connected. Only the case of compact $M$ can be found in the literature: If $M$ is orientable, the lemma follows from [23, Theorem 1, p. 11]. If the orientability is not supposed, define $f: M \rightarrow R^{2 n}$ by $f(x)=0$. Then $f$ is simply connected, i.e., $\pi_{0}(f)=\pi_{1}(f)=0$, where $\pi_{i}(f)=\pi_{i}\left(C_{f}, M\right)$ with $C_{f}$ the mapping cylinder of $f$. By [32, Theorem 7, p. 445], $f$ is homotopic to a locally flat embedding (even for $n \geqq 3$ ). Cf. also [12, Embedding Theorem 3] (for $n \geqq 2$ ). For the case where $M$ is non-compact a proof has been sketched by J. Dancis (written personal communication, 1980) and L. Siebenmann (oral personal communication, 1981).

\section{PL approximation of quasisymmetric embeddings in Euclidean spaces}

2.1. In this section we prove first the main result of the section, Theorem 2.16. Then we give two corollaries of it. These results concern PL approximation of quasisymmetric embeddings in Euclidean spaces. We close the section by a similar theorem about weakly quasisymmetric embeddings.

We begin by defining cubical decompositions.

2.2. Let $n \geqq 1$. For $k \in \boldsymbol{Z}$ let $\mathscr{L}_{n}(k)$ denote the family of closed $n$-cubes in $R^{n}$ with side length $2^{k}$ and with vertices in $2^{k} Z^{n}$. Let $\mathscr{L}_{n}=\cup\left\{\mathscr{L}_{n}(k) \mid k \in \boldsymbol{Z}\right\}$. For $Q \in \mathscr{L}_{n}$ let $z_{Q}$ denote the center and $2 \lambda_{Q}$ the side length of $Q$. Define $\alpha_{Q}: R^{n} \rightarrow R^{n}$ by $\alpha_{Q}(x)=$ $z_{Q}+\lambda_{Q} x$; then $Q=\alpha_{Q} I^{n}$.

2.3. Lemma. Let $X \subset R^{n}$ and let $Y$ be an open subset of $X$ such that $Y$ is the union of a subfamily of $\mathscr{L}_{n}$ which is locally finite in $Y$. Then there exists a subfamily $\chi$ of $\mathscr{L}_{n}$ with the following properties:

(1) $Y=\cup \chi$.

(2) If $Q, R \in \chi, Q \cap R \neq \emptyset$, and $Q \neq R$, then int $Q \cap \operatorname{int} R=\emptyset$ and

$$
\lambda_{Q} / \lambda_{R} \in\{1 / 2,1,2\} \text {. }
$$

(3) $\alpha_{Q} I^{n}(3) \cap X=\cup\left\{R \in \mathscr{L}_{n} \mid R \cap Q \neq \emptyset, \lambda_{R}=\lambda_{Q}, R \subset Y\right\}$ for $Q \in \chi$. 
Moreover, if $\varphi$ is a continuous map of $Y$ into a metric space $(Z, d)$ and if $\varepsilon \in C_{+}(Y)$, then $\chi$ can be chosen in such a way that

(4) $d(\varphi Q)<\varepsilon(x)$ if $x \in Q \in \chi$.

Proof. We construct $\chi$ which satisfies (1),.., (4). For $Q \in \mathscr{L}_{n}$ let $Q^{\prime}$ denote the right side of (3). Let

$$
\mathscr{L}=\left\{Q \in \mathscr{L}_{n} \mid Q \subset Y, \alpha_{Q} I^{n}(3) \cap X=Q^{\prime}, d\left(\varphi Q^{\prime}\right)<\min \varepsilon Q^{\prime}\right\} .
$$

Let $\chi_{0}=\mathscr{L} \cap \mathscr{L}_{n}(0)$. For $i \geqq 1$ we define $\chi_{i}$ inductively as the family of all cubes $Q \in \mathscr{L} \cap \mathscr{L}_{n}(-i)$ such that for no $j<i$ there is $R \in \chi_{j}$ containing $Q$. Define $\chi=\cup\left\{\chi_{i} \mid i \geqq 0\right\}$. To prove (1), let $x \in Y$. There are $i \geqq 0, k \geqq 1$, and $P_{1}, \ldots, P_{k} \in \mathscr{L}_{n}(-i)$ such that $P=P_{1} \cup \ldots \cup P_{k}$ is a neighborhood of $x$ in $Y$. For each $j>i$ there is $Q_{j} \in \mathscr{L}_{n}(-j)$ with $x \in Q_{j} \subset Y$. If $j$ is large enough, then $\alpha_{Q_{j}} I^{n}(3) \cap X \subset P$, which implies $\alpha_{Q_{j}} I^{n}(3) \cap X=Q_{j}^{\prime}$, and $d\left(\varphi Q_{j}^{\prime}\right)<\min \varepsilon Q_{j}^{\prime}$. Thus $Q_{j} \in \mathscr{L}$, whence $x \in \cup_{l=0}^{j}\left(\cup \chi_{l}\right)$. The first assertion in (2) is clear. In the second we may assume that $Q \in \chi_{i}$ and $R \in \chi_{j} \quad$ with $\quad j \geqq i . \quad$ Let $\quad \mathscr{S}=\left\{S \in \mathscr{L}_{n}(-i-1) \mid S \cap Q \neq \emptyset, \quad S \notin Q, \quad S \subset Y\right\} . \quad$ Then $\alpha_{Q}\left(2 I^{n} \backslash J^{n}\right) \cap X=\cup \mathscr{S}$. If $S \in \mathscr{S}$, we have $S^{\prime}=\alpha_{S} I^{n}(3) \cap X \subset Q^{\prime}$ and, hence, $d\left(\varphi S^{\prime}\right)<\min \varepsilon S^{\prime}$, which implies $S \in \mathscr{L}$. It follows that $j=i$ or $i+1$; consequently, $\lambda_{Q} / \lambda_{R} \in\{1,2\}$. The conditions (3) and (4) are satisfied by construction.

2.4. Let $X$ and $Y$ be as in 2.3 and let $\chi$ be any subfamily of $\mathscr{L}_{n}$ satisfying (1), ..., (3). Dividing each cube of $\chi$ into $2^{n}$ cubes by bisecting the sides we get a new cube family $\bar{\chi}$. Obviously, the conditions (1),.., (3) are satisfied also by $\bar{\chi}$. If $\chi$ satisfies (4), $\bar{\chi}$ satisfies it, too. A cube family $\bar{\chi}$ obtained in this manner is called a cubical decomposition of $Y$ with respect to $X$.

2.5. From now on and up to the end of the proof of 2.16, we suppose that $X$ and $Y$ are as in 2.3 and that $\mathscr{K}$ is a cubical decomposition of $Y$ with respect to $X$. Let $\varepsilon>0$ and let $f: X \rightarrow R^{q}$ be an $\eta$-quasisymmetric embedding, where $(n, q)$ is admissible. Finally, we assume that $Y$ is a manifold and thus a PL manifold.

For $Q \in \mathscr{K}$ we set $\varrho_{Q}=\left|f\left(z_{Q}\right)-f\left(z_{Q}+\lambda_{Q} e_{1}\right)\right|$ and $Q^{*}=\alpha_{Q} I^{n}(9 / 8) \cap X$. Then $Q^{*} \cap \alpha_{R} J^{n}(15 / 8)=\emptyset$ if $Q, R \in \mathscr{K}$ and $Q \cap R=\emptyset$.

2.6. Lemma. There are constants $c_{1} \geqq 1$ and $c_{2} \geqq 1$ depending only on $n$ and $\eta$ with the following properties:

(1) If $Q, R \in \mathscr{K}, Q \cap R \neq \emptyset$, and $Q \neq R$, then $\varrho_{Q} / c_{1} \leqq\left|f\left(z_{Q}\right)-f\left(z_{R}\right)\right| \leqq c_{1} \varrho_{Q}$.

(2) If $Q, R \in \mathscr{K}$ and $Q \cap R \neq \emptyset$, then $\varrho_{Q} / \varrho_{R} \leqq c_{1}^{2}$.

(3) If $Q \in \mathscr{K}, x \in Q^{*}$, and $y \in X \backslash \alpha_{Q} J^{n}(15 / 8)$, then $|f(x)-f(y)| \geqq \varrho_{Q} / c_{2}$.

Proof. (1): We have $(3 / 2) \lambda_{Q}<\left|z_{Q}-z_{R}\right| \leqq 3 \sqrt{n} \lambda_{Q}$. Hence

$$
\varrho_{Q} / \eta(2 / 3) \leqq\left|f\left(z_{Q}\right)-f\left(z_{R}\right)\right| \leqq \eta(3 \sqrt{n}) \varrho_{Q} .
$$

(2): This follows from (1). 
(3): Since $\quad(15 / 8) \lambda_{Q} \leqq\left|z_{Q}-y\right|$, we have $\varrho_{Q} \leqq \eta(8 / 15)\left|f\left(z_{Q}\right)-f(y)\right|$. Since $\left|z_{Q}-x\right| \leqq(9 / 8) \sqrt{n} \lambda_{Q} \leqq(3 / 2) \sqrt{n}|x-y|$ and thus $\left|z_{Q}-y\right| \leqq t|x-y|$, where $t=(3 / 2) \sqrt{n}+1$, we have $\left|f\left(z_{Q}\right)-f(y)\right| \leqq \eta(t)|f(x)-f(y)|$. Hence $\varrho_{Q} \leqq \eta(8 / 15) \eta(t)|f(x)-f(y)|$.

2.7. Let $x_{1}, x_{2}$, and $x$ be the least natural numbers such that

$$
2^{x_{1}} \geqq 2 \sqrt{q}, \quad 2^{x_{2}} \geqq 2 c_{1}^{2}, \quad \text { and } \quad x \geqq 2^{x_{1}+x_{2}}\left(2 c_{1}+\sqrt{q} 2^{x_{2}-x_{1}}\right) .
$$

These numbers depend only on $n, q$, and $\eta$. Define

$$
\begin{aligned}
& E_{1}=\left\{2^{k}|k \in \boldsymbol{Z},| k \mid \leqq x_{2}\right\}, \\
& E_{2}=\left\{2^{-x_{1}-x_{2}}\left(k_{1}, \ldots, k_{q}\right)\left|k_{i} \in \boldsymbol{Z},\right| k_{i} \mid \leqq x\right\} .
\end{aligned}
$$

For each $Q \in \mathscr{K}$ let $\mu_{Q}$ be the integer with $2^{\mu_{Q} \leqq} \varrho_{Q}<2^{\mu_{Q}+1}$, and define $s_{Q}=2^{\mu_{Q}}$. Then $\varrho_{Q} / 2<s_{Q} \leqq \varrho_{Q}$. We choose $b_{Q} \in 2^{\mu_{Q}-x_{1}} Z^{q}$ such that $\left|b_{Q}-f\left(z_{Q}\right)\right| \leqq \sqrt{q} 2^{\mu_{Q}-x_{1}-1}$ $\left(\leqq s_{Q} / 4\right)$. The following lemma can now be proved by the aid of 2.6(1) and 2.6(2) exactly as [18, Lemma 2.4$]$.

2.8. Lemma. If $Q, R \in \mathscr{K}, Q \cap R \neq \emptyset$, then

(1) $s_{Q} / s_{R} \in E_{1}$,

(2) $\left(b_{Q}-b_{R}\right) / s_{Q} \in E_{2}$.

2.9. We express $\mathscr{K}$ as a disjoint union $\mathscr{K}=\mathscr{K}_{1} \cup \ldots \cup \mathscr{K}_{M}$, where each family $\mathscr{K}_{i}$ is disjoint and where $M=M(n)$. In fact, this can be done with $M(n)=2^{n}$ as follows. Number the cubes of $\mathscr{L}_{n}(0)$ in $I^{n}$ by $S_{1}, \ldots, S_{M(n)}$. Let $\mathscr{K}=\bar{\chi}$. If $Q \in \mathscr{K}$, let $R$ be the unique cube in $\chi$ with $\lambda_{R}=2 \lambda_{Q}$ and $Q \subset R$; then set $S_{Q}=\alpha_{R}^{-1} Q$. Define $\mathscr{K}_{i}=\left\{Q \in \mathscr{K} \mid S_{Q}=S_{i}\right\}$. It is obvious that the families $\mathscr{K}_{1}, \ldots, \mathscr{K}_{M(n)}$ satisfy the requirements. We set $\mathscr{K}_{i}^{*}=\mathscr{K}_{1} \cup \ldots \cup \mathscr{K}_{i}$ and $F_{i}=\cup\left\{Q^{*} \mid Q \in \mathscr{K}_{i}^{*}\right\}$.

2.10. Let $\mathscr{I}_{n}=\left\{Q \in \mathscr{L}_{n}(0) \mid Q \subset 2 I^{n} \backslash J^{n}\right\}$. If $Q \in \mathscr{I}_{n}$ and $t \in\{1,2,3\}$, we set $Q(t)=\alpha_{Q} I^{n}\left(1+2^{-t}\right)$. Let $\mathscr{T}_{n}$ be the finite set consisting of manifolds which can be expressed as a union $I^{n} \cup(\cup \mathscr{I}), \mathscr{I} \subset \mathscr{I}_{n}$. If $T \in \mathscr{T}_{n}$, let $\mathscr{P}(T)$ denote the finite set of pairs $\left(P^{\prime}, P\right)$ of the form

$$
\begin{aligned}
& P^{\prime}=\left(I^{n}(9 / 8) \cup Q_{1}\left(t_{1}\right) \cup \ldots \cup Q_{k}\left(t_{k}\right)\right) \cap T, \\
& P=\left(Q_{1}\left(t_{1}\right) \cup \ldots \cup Q_{k}\left(t_{k}\right)\right) \cap T,
\end{aligned}
$$

where $Q_{i} \in \mathscr{I}_{n}, Q_{i} \subset T$, and $t_{i} \in\{1,2,3\}$ for $i \leqq k \geqq 0$. It is obvious that the sets $P^{\prime}, P$, and $\overline{P^{\prime} \backslash P}$ are PL $n$-manifolds and that $P \cap \overline{P^{\prime} \backslash P}$ is a PL $(n-1)$-manifold.

Let $1 \leqq i \leqq M(n)$ and $Q \in \mathscr{K}_{i}$. Since $Y$ is a manifold, we have $T_{Q}=I^{n}(2) \cap$ $\alpha_{Q}^{-1} X \in \mathscr{T}_{n}$. We set

$$
\begin{aligned}
& P_{Q}=\left(\cup\left\{\alpha_{Q}^{-1} R^{*} \mid R \in \mathscr{K}_{i-1}^{*}, R \cap Q \neq \emptyset\right\}\right) \cap T_{Q}, \\
& P_{Q}^{\prime}=\alpha_{Q}^{-1} Q^{*} \cup P_{Q} .
\end{aligned}
$$

Then $\left(P_{Q}^{\prime}, P_{Q}\right) \in \mathscr{P}\left(T_{Q}\right)$. 
2.11. Let $T \in \mathscr{T}_{n}$, let $g: T \rightarrow R^{q}$ be an embedding, and $\varepsilon_{0}>0$. Let

$$
\left(P^{\prime}, P\right) \in \mathscr{P}(T) \text {. }
$$

Then by 1.9 (or also by 1.10 .2 if $n=q=2$ or 3 ) there exists $\delta \in\left(0, \varepsilon_{0}\right]$ such that if $g_{1}: P \rightarrow R^{q}$ is a PL embedding with $d\left(g_{1}, g \mid P\right)<\delta$, there is a PL embedding $g_{1}^{*}: P^{\prime} \rightarrow R^{q}$ with $g_{1}^{*} \mid P=g_{1}$ and $d\left(g_{1}^{*}, g \mid P^{\prime}\right)<\varepsilon_{0}$. We let $\delta\left(g, \varepsilon_{0}\right)$ denote the greatest $\delta$ satisfying this for all $\left(P^{\prime}, P\right) \in \mathscr{P}(T)$.

2.12. For $T \in \mathscr{T}_{n}$ let $H_{\eta}(T)$ be the set of all $\eta$-quasisymmetric embeddings $g: T \rightarrow R^{q}$ with $|g(0)| \leqq 1 / 4$ and $3 / 4 \leqq\left|g\left(e_{1}\right)\right| \leqq 3$. In the topology of uniform convergence $H_{\eta}(T)$ is compact by [38, Remark 3.6 and Theorem 3.7]. Let $H_{\eta}=$ $\cup\left\{H_{\eta}(T) \mid T \in \mathscr{T}_{n}\right\}$. We set $\delta^{*}\left(\varepsilon_{0}\right)=\inf \left\{\delta\left(g, \varepsilon_{0}\right) \mid g \in H_{\eta}\right\} \quad\left(\leqq \varepsilon_{0}\right)$ for $\varepsilon_{0}>0$. Then as in [41, Lemma 2.6], we get $\delta^{*}\left(\varepsilon_{0}\right)>0$, and $\delta^{*}\left(\varepsilon_{0}\right)$ depends only on $n, q, \eta$, and $\varepsilon_{0}$.

For each $Q \in \mathscr{K}$ we define $\beta_{Q}: R^{q} \rightarrow R^{q}$ by $\beta_{Q}(x)=\left(x-b_{Q}\right) / s_{Q}$ and set $f_{Q}=$ $\beta_{Q} f \alpha_{Q} \mid T_{Q}$. Then $f_{Q} \in H_{\eta}$, because $\alpha_{Q}$ and $\beta_{Q}$ are similarities and because $\left|f_{Q}(0)\right|=$ $\left|f\left(z_{Q}\right)-b_{Q}\right| / s_{Q} \leqq 1 / 4,\left|f_{Q}\left(e_{1}\right)\right| \leqq \varrho_{Q} / s_{Q}+1 / 4<9 / 4$, and $\left|f_{Q}\left(e_{1}\right)\right| \geqq \varrho_{Q} / s_{Q}-1 / 4 \geqq 3 / 4$.

2.13. We choose positive numbers $\delta_{1} \leqq \ldots \leqq \delta_{M(n)}$ such that

$$
\delta_{M(n)}<\min \left(\varepsilon / 2,1 / 4 c_{1}^{2} c_{2}, 1 / 4 c_{1}^{2} \eta(2) \eta(2 \sqrt{n})\right)
$$

and $\delta_{i}<\delta^{*}\left(\delta_{i+1}\right) /\left(2^{\varkappa_{2}+1}+1\right)$. The numbers $\delta_{i}$ depend only on $n, q$, $\eta$, and $\varepsilon$.

2.14. Lemma. For every $i \in\{1, \ldots, M(n)\}$ there exist a finite set $A_{i}$ of $\mathrm{PL}$ embeddings $g: I^{n}(9 / 8) \cap T \rightarrow R^{q}$, where $T \in \mathscr{T}_{n}$, such that $A_{i}$ depends only on $n, q, \eta$, and $\varepsilon$, and an injective PL map $\varphi_{i}: F_{i} \rightarrow R^{q}$ with

(1) $\beta_{Q} \varphi_{i} \alpha_{Q} \mid \alpha_{Q}^{-1} Q^{*} \in A_{i}$ if $Q \in \mathscr{K}_{i}^{*}$,

(2) $d\left(f\left|Q^{*}, \varphi_{i}\right| Q^{*}\right) \leqq 2 \delta_{i} s_{Q}$ if $Q \in \mathscr{K}_{i}^{*}$.

Proof. The proof is by induction on $i$. Let $T \in \mathscr{T}_{n}$. We choose a finite subset $H\left(T, \delta_{1}\right) \subset H_{\eta}(T)$ such that for every $h \in H_{\eta}(T)$ there is $h^{\prime} \in H\left(T, \delta_{1}\right)$ with $d\left(h^{\prime}, h\right) \leqq \delta_{1}$. By 1.7 we choose for every $h \in H\left(T, \delta_{1}\right)$ a PL embedding $g_{h}: I^{n}(9 / 8) \cap$ $T \rightarrow R^{q}$ with $d\left(g_{h}, h \mid I^{n}(9 / 8) \cap T\right) \leqq \delta_{1}$. We define $A_{1}=\left\{g_{h} \mid h \in H\left(T, \delta_{1}\right), T \in \mathscr{T}_{n}\right\}$. If $Q \in \mathscr{K}$, we choose $h_{Q} \in H\left(T_{Q}, \delta_{1}\right)$ with $d\left(h_{Q}, f_{Q}\right) \leqq \delta_{1}$ and set $g_{Q}=g_{h_{Q}}$.

We define a PL map $\varphi_{1}: F_{1} \rightarrow R^{q}$ by $\varphi_{1}\left|Q^{*}=\beta_{Q}^{-1} g_{Q} \alpha_{Q}^{-1}\right| Q^{*}$ for $Q \in \mathscr{K}_{1}$. Then (1) is satisfied. We have $d\left(f\left|Q^{*}, \varphi_{1}\right| Q^{*}\right)=s_{Q} d\left(f_{Q} \mid \alpha_{Q}^{-1} Q^{*}, g_{Q}\right) \leqq 2 \delta_{1} s_{Q}$ if $Q \in \mathscr{K}_{1}$. If $Q, R \in \mathscr{K}_{1}, Q \neq R, s_{Q} \geqq s_{R}, x \in Q^{*}$, and $y \in R^{*}$, then, since $y \notin \alpha_{Q} J^{n}(15 / 8)$, we get by $2.6(3)$

$$
\begin{aligned}
\left|\varphi_{1}(x)-\varphi_{1}(y)\right| & \geqq|f(x)-f(y)|-\left|f(x)-\varphi_{1}(x)\right|-\left|f(y)-\varphi_{1}(y)\right| \\
& \geqq \varrho_{Q} / c_{2}-2 \delta_{1}\left(s_{Q}+s_{R}\right) \geqq s_{Q}\left(1 / c_{2}-4 \delta_{1}\right)>0 .
\end{aligned}
$$

Hence $\varphi_{1}$ is injective.

Suppose now that $A_{i-1}$ and $\varphi_{i-1}$ satisfying (1) and (2) have been constructed. Let $Q \in \mathscr{K}_{i}$. Consider the PL embedding $\gamma_{Q}=\beta_{Q} \varphi_{i-1} \alpha_{Q} \mid P_{Q}$. If $x \in P_{Q}$, there is $R \in \mathscr{K}_{i-1}^{*}$ such that $\alpha_{Q}(x) \in R^{*}$ and $R \cap Q \neq \emptyset$. Then $\gamma_{Q}(x)=\beta_{Q} \beta_{R}^{-1} \psi \alpha_{R}^{-1} \alpha_{Q}(x)$, 
where $\psi=\beta_{R} \varphi_{i-1} \alpha_{R} \mid \alpha_{R}^{-1} R^{*} \in A_{i-1}$. It is easy to see that the maps $\alpha_{R}^{-1} \alpha_{Q}$ belong to a finite set depending only on $n$. The sets $I^{n}(2) \cap \alpha_{Q}^{-1} R^{*}$ are among the sets $P$ considered in 2.10. Thus the sets $P_{Q} \cap \alpha_{Q}^{-1} R^{*}$ belong to a finite set depending only on $n$. Since $\beta_{Q} \beta_{R}^{-1}(y)=s_{R} y / s_{Q}+\left(b_{R}-b_{Q}\right) / s_{Q}$, by 2.8 the maps $\beta_{Q} \beta_{R}^{-1}$ belong to a finite set depending only on $n, q$, and $\eta$. Hence the maps $\gamma_{Q}$ belong to a finite set $C_{i}$ depending only on $n, q, \eta$, and $\varepsilon$. If $x$ and $R$ are as above, we have

$$
\begin{aligned}
\left|\gamma_{Q}(x)-f_{Q}(x)\right| & =\left|\varphi_{i-1}\left(\alpha_{Q}(x)\right)-f\left(\alpha_{Q}(x)\right)\right| / s_{Q} \\
& \leqq 2 \delta_{i-1} s_{R} / s_{Q} \leqq 2^{\varkappa_{2}+1} \delta_{i-1}
\end{aligned}
$$

by 2.8 , which implies that $d\left(\gamma_{Q}, h_{Q} \mid P_{Q}\right)<\delta^{*}\left(\delta_{i}\right)$. Hence there is a PL embedding $g_{Q}^{*}: P_{Q}^{\prime} \rightarrow R^{q}$ with $g_{Q}^{*} \mid P_{Q}=\gamma_{Q}$ and $d\left(g_{Q}^{*}, h_{Q} \mid P_{Q}^{\prime}\right)<\delta_{i}$. We can choose the maps $g_{Q}^{*}$ such that the maps $g_{Q}^{*} \mid \alpha_{Q}^{-1} Q^{*}$ belong to a finite set $G_{i}$, depending only on $n, q$, $\eta$, ar. $\varepsilon$, of PL embeddings $g: I^{n}(9 / 8) \cap T \rightarrow R^{q}, T \in \mathscr{T}_{n}$.

We set $\varphi_{i} \mid F_{i-1}=\varphi_{i-1}$ and $\varphi_{i}\left|Q^{*}=\beta_{Q}^{-1} g_{Q}^{*} \alpha_{Q}^{-1}\right| Q^{*}$ for $Q \in \mathscr{K}_{i}$. Then $\varphi_{i}: F_{i} \rightarrow R^{q}$ is a well-defined PL map and (1) is satisfied with $A_{i}=A_{i-1} \cup G_{i}$. If $Q \in \mathscr{K}_{i}$, then $\left.d_{1}\left|2^{*}, \varphi_{i}\right| Q^{*}\right)=s_{Q} d\left(f_{Q}\left|\alpha_{Q}^{-1} Q^{*}, g_{Q}^{*}\right| \alpha_{Q}^{-1} Q^{*}\right) \leqq 2 \delta_{i} s_{Q}$, which implies (2). We prove that $\varphi$ is injective. Observe first that $\varphi_{i}$ is the embedding $\beta_{Q}^{-1} g_{Q}^{*} \alpha_{Q}^{-1}$ on $\alpha_{Q} P_{Q}^{\prime}$ in every $Q \in \mathscr{K}_{i}$. Thus it suffices to show that $\Delta=\left|\varphi_{i}(x)-\varphi_{i}(y)\right|>0$ if $Q \in \mathscr{K}_{i}$, $R \in \mathscr{K}_{i}^{*}, x \in Q^{*}, y \in R^{*}$, and either (a) $Q \cap R=\emptyset$ or (b) $Q \cap R \neq \emptyset$ and $y \notin \alpha_{Q} I^{n}(2)$. We proceed as in (2.15). If either (a) holds or (b) holds with $s_{Q} \geqq s_{R}$, we get $\Delta \geqq$ $s_{Q}\left(1 / c_{2}-4 \delta_{i}\right)>0$. If (b) holds with $s_{Q}<s_{R}$, we use the fact $\varrho_{Q} \geqq \varrho_{R} / c_{1}^{2}$, implied by $2.6(2)$, and get $\Delta \geqq s_{R}\left(1 / c_{1}^{2} c_{2}-4 \delta_{i}\right)>0$.

2.16. Theorem. Let $(n, q)$ be admissible, let $\eta: R_{+}^{1} \rightarrow R_{+}^{1}$ be a homeomorphism, and let $\varepsilon>0$. Then there exist a homeomorphism $\eta^{*}: R_{+}^{1} \rightarrow R_{+}^{1}$ and a finite set $D$ of $\mathrm{PL}$ embeddings $g: T \rightarrow R^{q}, T \in \mathscr{T}_{n}$, with the following property: Let $X \subset R^{n}$, let $f: X \rightarrow R^{q}$ be an $\eta$-quasisymmetric embedding, let $Y$ be an open subset of $X$ which $i s$ the union of a subfamily of $\mathscr{L}_{n}$ locally finite in $Y$ and which is a manifold, and let $\mathscr{H}$ be a cubical decomposition of $Y$ with respect to $X$. Then there exists an $\eta^{*}$-quasisymmetric embedding $f^{*}: X \rightarrow R^{q}$ such that

(1) $f^{*}|X \backslash Y=f| X \backslash Y$,

(2) $f^{*} \mid Y$ is $\mathrm{PL}$,

(3) $d\left(f^{*}|Q, f| Q\right) \leqq \varepsilon \varrho_{Q}$ for every $Q \in \mathscr{K}$,

(4) $\beta_{Q} f^{*} \alpha_{Q} \mid T_{Q} \in D$ for every $Q \in \mathscr{K}$.

Proof. We define $f^{*}=\varphi_{M(n)} \cup(f \mid X \backslash Y)$. Then (1), (2), and (3) are satisfied. One can find $D$ and prove (4) in the same way as one obtained the relation $\gamma_{Q} \in C_{i}$ in the proof of 2.14. Two auxiliary results will be proved next. The first one, (2.17), implies that $f^{*}$ is an embedding.

Let $M=M(n)$. Define $a_{0}=4 \delta_{M} c_{1}^{2} c_{2} \in(0,1)$ and $a=\left(1-a_{0}\right)^{-1}$. We show that

$$
|f(x)-f(y)| / a \leqq\left|f^{*}(x)-f^{*}(y)\right| \leqq a|f(x)-f(y)|
$$


if $x \in Q \in \mathscr{K}$ and $y \in X \backslash \alpha_{Q} J^{n}(2)$. Let $\Delta=|| f^{*}(x)-f^{*}(y)|-| f(x)-f(y) \|$. Suppose first that $y \in R \in \mathscr{K}$. Then $\Delta \leqq 2 \delta_{M}\left(s_{Q}+s_{R}\right)$. If $Q \cap R \neq \emptyset$, this implies, by 2.6, $\Delta \leqq 2 \delta_{M}\left(1+c_{1}^{2}\right) \varrho_{Q} \leqq 2 \delta_{M}\left(1+c_{1}^{2}\right) c_{2}|f(x)-f(y)|$, whereas if $Q \cap R=\emptyset$, then $\Delta \leqq$ $4 \delta_{M} c_{2}|f(x)-f(y)|$. Suppose now that $y \in X \backslash Y$. Then $\Delta \leqq 2 \delta_{M} \varrho_{Q} \leqq 2 \delta_{M} c_{2}|f(x)-f(y)|$. Thus $\Delta \leqq a_{0}|f(x)-f(y)|$ in all cases. Hence (2.17) holds.

Define $b_{0}=4 \delta_{M} c_{1}^{2} \eta(2) \eta(2 \sqrt{n}) \in(0,1)$ and $b=\left(1-b_{0}\right)^{-1}$. We show that

$$
|f(x)-f(y)| / b \leqq\left|f^{*}(x)-f^{*}(y)\right| \leqq b|f(x)-f(y)|
$$

if $Q \in \mathscr{K}, x, y \in \alpha_{Q} I^{n}(2) \cap X$, and $|x-y| \geqq \lambda_{Q}$. Let $\Delta$ te as above. Then $\Delta \leqq 4 \delta_{M} c_{1}^{2} \varrho_{Q}$. We may assume that $\left|x-z_{Q}\right| \geqq \lambda_{Q} / 2$. Then $\varrho_{Q} \leqq \eta(2)\left|f(x)-f\left(z_{Q}\right)\right|$. Since $\left|x-z_{Q}\right| \leqq$ $2 \sqrt{n} \lambda_{Q} \leqq 2 \sqrt{n}|x-y|$, we have $\left|f(x)-f\left(z_{Q}\right)\right| \leqq \eta(2 \sqrt{n})|f(x)-f(y)|$. Thus $\Delta \leqq$ $b_{0}|f(x)-f(y)|$, whence (2.18).

Now we prove that $f^{*}$ is quasisymmetric. Since every $g \in D$ is quasisymmetric and since $\alpha_{Q}$ and $\beta_{Q}$ are similarities, (4) implies that $f^{*} \mid \alpha_{Q} I^{n}(2) \cap X$ is $\eta_{0}$-quasisymmetric for every $Q \in \mathscr{K}$ for some $\eta_{0}$ depending only on $n, q$, $\eta$, and $\varepsilon$. Set $\eta_{1}(t)=$ $\sup \left\{\eta_{0}(s) \eta\left(s^{\prime}\right) \mid s, s^{\prime} \in I, s^{\prime} \leqq t\right\}$ for $t \in I$. Then $\eta_{1}: I \rightarrow R_{+}^{1}$ is bounded and nondecreasing. If $\varepsilon_{1}>0$, there is $\delta \in(0,1)$ such that $\eta_{0}(\delta) \leqq \varepsilon_{1} / \eta(1)$ and $\eta(\delta) \leqq$ $\varepsilon_{1} / \eta_{0}(1)$, whence $\eta_{1}\left(\delta^{2}\right) \leqq \varepsilon_{1}$. Thus $\eta_{1}(t) \rightarrow 0$ as $t \rightarrow 0$. It follows that there is a homeomorphism $\eta_{2}: R_{+}^{1} \rightarrow R_{+}^{1}$ with $\eta_{1} \leqq \eta_{2} \mid I$.

Let $u, v, x \in X, v \neq x, t=|u-x| /|v-x|$, and $t^{\prime}=\left|f^{*}(u)-f^{*}(x)\right| /\left|f^{*}(v)-f^{*}(x)\right|$. We need an estimate $t^{\prime} \leqq \eta^{*}(t)$. We divide the consideration into four separate cases.

Case 1. Let $x \in Q \in \mathscr{K}$ and $u, v \in \alpha_{Q} I^{n}(2)$. Then $t^{\prime} \leqq \eta_{0}(t)$.

Case 2. Let $x \in Q \in \mathscr{K}, u \in \alpha_{Q} I^{n}(2)$, and $v \notin \alpha_{Q} I^{n}(2)$.

Subcase $2 \mathrm{a}:|u-x| \geqq \lambda_{Q}$. Then, by (2.18) and (2.17),

$$
\begin{aligned}
\left|f^{*}(u)-f^{*}(x)\right| & \leqq b|f(u)-f(x)| \leqq b \eta(t)|f(v)-f(x)| \\
& \leqq a b \eta(t)\left|f^{*}(v)-f^{*}(x)\right| .
\end{aligned}
$$

Subcase $2 \mathrm{~b}:|u-x|<\lambda_{Q}$. Choose $y \in Q$ with $|x-y|=\lambda_{Q}$. Then $|u-x|<$ $|y-x|<|v-x|$. Hence by Subcase 2a,

$$
\begin{aligned}
t^{\prime} & =\frac{\left|f^{*}(u)-f^{*}(x)\right|}{\left|f^{*}(y)-f^{*}(x)\right|} \cdot \frac{\left|f^{*}(y)-f^{*}(x)\right|}{\left|f^{*}(v)-f^{*}(x)\right|} \\
& \leqq \eta_{0}\left(\frac{|u-x|}{|y-x|}\right) a b \eta\left(\frac{|y-x|}{|v-x|}\right) \leqq a b \eta_{2}(t) .
\end{aligned}
$$

Case 3. Let $x \in Q \in \mathscr{K}, u \notin \alpha_{Q} I^{n}(2)$, and $v \in \alpha_{Q} I^{n}(2)$.

Subcase 3a: $|v-x| \geqq \lambda_{Q}$. Now

$$
\begin{aligned}
\left|f^{*}(u)-f^{*}(x)\right| & \leqq a|f(u)-f(x)| \leqq a \eta(t)|f(v)-f(x)| \\
& \leqq a b \eta(t)\left|f^{*}(v)-f^{*}(x)\right| .
\end{aligned}
$$


Subcase 3b: $|v-x|<\lambda_{Q}$. Choose $y \in Q$ with $|x-y|=\lambda_{Q}$. Then by Subcase $3 \mathrm{a}$ and since $|v-x|<|y-x|<|u-x|$,

$$
\begin{aligned}
t^{\prime} & =\frac{\left|f^{*}(u)-f^{*}(x)\right|}{\left|f^{*}(y)-f^{*}(x)\right|} \cdot \frac{\left|f^{*}(y)-f^{*}(x)\right|}{\left|f^{*}(v)-f^{*}(x)\right|} \\
& \leqq a b \eta\left(\frac{|u-x|}{|y-x|}\right) \eta_{0}\left(\frac{|y-x|}{|v-x|}\right) \leqq a b \eta(t) \eta_{0}(t) .
\end{aligned}
$$

Case 4. Let either $x \in Q \in \mathscr{K}$ and $u, v \notin \alpha_{Q} I^{n}(2)$ or $x \in X \backslash Y$. Then by (2.17),

$$
\begin{aligned}
\left|f^{*}(u)-f^{*}(x)\right| & \leqq a|f(u)-f(x)| \leqq a \eta(t)|f(v)-f(x)| \\
& \leqq a^{2} \eta(t)\left|f^{*}(v)-f^{*}(x)\right| .
\end{aligned}
$$

Thus there exists a homeomorphism $\eta^{*}: R_{+}^{1} \rightarrow R_{+}^{1}$ which depends only on $n, q, \eta$, and $\varepsilon$ such that $t^{\prime} \leqq \eta^{*}(t)$, i.e., such that $f^{*}$ is $\eta^{*}$-quasisymmetric.

2.19. Remark. In Theorem 2.16 we assumed that $Y$ is a manifold only in order to be able to use the PL approximation results 1.7 and 1.9. However, for $q \geqq n=1$ this assumption is redundant, and for $n \geqq 2, q \geqq n+3$ we could omit it by 1.10 .3 (cf. also 1.10.1). This remark also applies to 2.20 and 3.2.

2.20. Corollary. Let $n, q$, and $\eta$ be as in 2.16. Then there exists a homeomorphism $\eta^{*}: R_{+}^{1} \rightarrow R_{+}^{1}$ with the following property: Let $X, f$, and $Y$ be as in 2.16 and let $\varepsilon \in C_{+}(Y)$. Then there exists an $\eta^{*}$-quasisymmetric embedding $f^{*}: X \rightarrow R^{q}$ such that

(1) $f^{*}|X \backslash Y=f| X \backslash Y$,

(2) $f^{*} \mid Y$ is $\mathrm{PL}$,

(3) $\left|f^{*}(x)-f(x)\right|<\varepsilon(x)$ for every $x \in Y$.

Proof. This follows from 2.16 (choose $\varepsilon=1$ ) and 2.3 .

2.21. Corollary. Let $(n, q)$ be admissible and let $\eta: R_{+}^{1} \rightarrow R_{+}^{1}$ be a homeomorphism. Then there exists a homeomorphism $\eta^{*}: R_{+}^{1} \rightarrow R_{+}^{1}$ with the following property: Let $U \subset R^{n}$ be open, let $f: U \rightarrow R^{q}$ be an $\eta$-quasisymmetric embedding, and let $\varepsilon \in C_{+}(U)$. Then there exists an $\eta^{*}$-quasisymmetric PL embedding $g: U \rightarrow R^{q}$ such that $|g(x)-f(x)|<\varepsilon(x)$ for every $x \in U, \bar{g}|\partial U=\bar{f}| \partial U$, and, if $1 \leqq n=q \leqq 3$, $g U=f U$. Here $\bar{f}$ and $\bar{g}$ are the closed embeddings $\bar{U} \rightarrow R^{q}$ extending $f$ and $g$, respectively (cf. 1.2).

Proof. Let $\eta^{*}$ be the homeomorphism of 2.20. It is easy to see that $U$ is the union of a subfamily of $\mathscr{L}_{n}$ which is locally finite in $U$. Thus 2.20 gives an $\eta^{*}$-quasisymmetric PL embedding $g: U \rightarrow R^{q}$ with $|g(x)-f(x)|<\varepsilon(x)$ and $|g(x)-f(x)|<$ $d(f(x), \bar{f} \partial U)$ for every $x \in U$. Then $\bar{g}|\partial U=\bar{f}| \partial U$. Suppose that $n=q$. If $V$ is a component of $U$, we have $\partial g V=\bar{g} \partial V=\bar{f} \partial V=\partial f V$ and $g V \subset f V$, whence $g V=f V$. Thus $g U=f U$. 
2.22. Theorem. Let $(n, q)$ be admissible and let $H \geqq 1$. Then there exists $H^{*} \geqq 1$ with the following property: Let $X \subset R^{n}$, let $f: X \rightarrow R^{q}$ be a weakly $H$-quasisymmetric embedding, let $U \subset X$ be open in $R^{n}$, and let $\varepsilon \in C_{+}(U)$. Then there exists a weakly $H^{*}$-quasisymmetric embedding $f^{*}: X \rightarrow R^{q}$ such that

(1) $f^{*}|X \backslash U=f| X \backslash U$,

(2) $f^{*} \mid U$ is $\mathrm{PL}$,

(3) $\left|f^{*}(x)-f(x)\right|<\varepsilon(x)$ for every $x \in U$,

(4) $\bar{f}^{*}|\partial U=\bar{f}| \partial U$, where $\bar{f}$ and $\bar{f}^{*}$ are the closed embeddings $\bar{U} \rightarrow R^{q}$ extending $f \mid U$ and $f^{*} \mid U$, respectively (cf. 1.2 ),

(5) $f^{*} U=f U$ if $1 \leqq n=q \leqq 3$.

Proof. It suffices to prove the modification of Theorem 2.16 in which $\eta$ is replaced by $H, \eta^{*}$ is replaced by $H^{*}$, the embedding $f$ is only assumed to be weakly $H$-quasisymmetric, $Y$ is assumed to be open in $R^{n}$, and the embedding $f^{*}$ is only required to be weakly $H^{*}$-quasisymmetric. To this end, we modify the proof of 2.16 (in $2.5-2.18$ ). We first observe that in this proof, with two exceptions, one actually used, instead of the assumption that the embedding $f$ is $\eta$-quasisymmetric, only the assumption that $f \mid Q^{\prime}$ is $\eta$-quasisymmetric for every $Q \in \mathscr{K}$, where $Q^{\prime}=$ $\alpha_{Q} I^{n}(3) \cap X$. The exceptions were (a) the proof of 2.6(3) and (b) the estimation of $t^{\prime}$ in Cases 1-4. Now let $f$ and $Y$ be as in the present modification of 2.16 , and let $\mathscr{K}$ be a cubical decomposition of $Y$ with respect to $X$. Then $Q^{\prime}=\alpha_{2} I^{n}(3) \subset Y$ for every $Q \in \mathscr{K}$. It follows from [38, Theorem 2.16] that $f \mid Q^{\prime}$ is $\eta$-quasisymmetric for every $Q \in \mathscr{K}$ with $\eta$ depending only on $n, q$, and $H$. Hence it suffices to reexamine (a) and (b).

For (a) let $Q \in \mathscr{K}, x \in Q^{*}$, and $y \in X \backslash \alpha_{Q} J^{n}(15 / 8)$. Choose $u \in \partial \alpha_{Q} J^{n}(15 / 8) \subset X$ with $|x-u| \leqq|x-y|$. Then $|f(x)-f(u)| \leqq H|f(x)-f(y)|$. On the other hand, the proof of 2.6(3) shows that $|f(x)-f(u)| \geqq \varrho_{Q} / c$, where $c=\eta(8 / 15) \eta((3 / 2) \sqrt{n}+1)$. Therefore $\varrho_{Q} \leqq H c|f(x)-f(y)|$. Hence 2.6(3) holds.

For (b) suppose that $t \leqq 1$. Then, proceeding as earlier, we get $t^{\prime} \leqq \eta_{0}(1)$ in Case $1, t^{\prime} \leqq a b H$ in Subcases $2 \mathrm{a}$ and $3 \mathrm{a}, t^{\prime} \leqq \eta_{0}(1) a b H$ in Subcase $2 \mathrm{~b}$, and $t^{\prime} \leqq a^{2} H$ in Case 4; Subcase 3b does not occur. This implies that the embedding $f^{*}$ is weakly $H^{*}$-quasisymmetric with $H^{*}$ depending only on $n, q, H$, and $\varepsilon$.

\section{PL approximation of bilipschitz embeddings in Euclidean spaces}

3.1. In this section we apply 2.16 to proving bilipschitz analogues of 2.20 and 2.21. Both [41, Theorem 2.4] and [18, Theorem 3.1] are special cases of Corollary 3.3 for $1 \leqq n=q \leqq 3$.

3.2. Theorem. Let $(n, q)$ be admissible and let $L \geqq 1$. Then there exists $L^{*} \geqq 1$ with the following property: Let $X \subset R^{n}$, let $f: X \rightarrow R^{q}$ be an L-bilipschitz 
embedding, let $Y$ be an open subset of $X$ which is the union of a subfamily of $\mathscr{L}_{n}$ locally finite in $Y$ and which is a manifold, and let $\varepsilon \in C_{+}(Y)$. Then there exists an $L^{*}$-bilipschitz embedding $f^{*}: X \rightarrow R^{q}$ such that

(1) $f^{*}|X \backslash Y=f| X \backslash Y$,

(2) $f^{*} \mid Y$ is PL,

(3) $\left|f^{*}(x)-f(x)\right|<\varepsilon(x)$ for every $x \in Y$.

Proof. Every $L$-bilipschitz embedding is $\eta_{L}$-quasisymmetric, $\eta_{L}(t)=L^{2} t$. Let $D$ be the finite set of PL embeddings $g: T \rightarrow R^{q}, T \in \mathscr{T}_{n}$, given by 2.16 with substitutions $(n, q) \mapsto(n, q), \eta \mapsto \eta_{L}, \varepsilon \mapsto 1 / 3 L^{2}$. Let now $X, f, Y$, and $\varepsilon$ be as in 3.2. By 2.3 there is a cubical decomposition $\mathscr{K}$ of $Y$ with respect to $X$ such that $\lambda_{Q} \leqq \varepsilon(x)$ if $x \in Q \in \mathscr{K}$. Then 2.16 gives an emtedding $f^{*}: X \rightarrow R^{q}$ such that (1) and (2) hold and such that $d\left(f^{*}|Q, f| Q\right) \leqq \varrho_{Q} / 3 L^{2} \leqq \lambda_{Q} / 3 L$ and $\beta_{Q} f^{*} \alpha_{Q} \mid T_{Q} \in D$ for every $Q \in \mathscr{K}$. It follows that also (3) holds.

There is $L_{0} \geqq 1$ depending only on $n, q$, and $L$ such that each $g \in D$ is $L_{0}$-bilipschitz. We show that $f^{*}$ is $L^{*}$-bilipschitz with $L^{*}=\max \left(2 L L_{0}, 3 L\right)$. Let $x, y \in X$, $x \neq y$, and set $\Delta=\left|f^{*}(x)-f^{*}(y)\right|, \delta=|x-y|$. If $x, y \in X \backslash Y$, then $1 / L \leqq \Delta / \delta \leqq L$. If $x, y \in \alpha_{Q} I^{n}(2)$ for some $Q \in \mathscr{K}$, then $\Delta / \delta \leqq s_{Q} L_{0} / \lambda_{Q} \leqq L L_{0}$ and $\Delta / \delta \geqq s_{Q} / L_{0} \lambda_{Q} \geqq$ $1 / 2 L L_{0}$. If $x \in Q \in \mathscr{K}, y \in R \in \mathscr{K}, x \notin \alpha_{R} I^{n}(2)$, and $y \notin \alpha_{Q} I^{n}(2)$, then $\lambda_{Q}, \lambda_{R} \leqq \delta$, whence $\Delta \leqq|f(x)-f(y)|+\left(\lambda_{Q}+\lambda_{R}\right) / 3 L \leqq(L+2 / 3 L) \delta$ and

$$
\Delta \geqq|f(x)-f(y)|-\left(\lambda_{Q}+\lambda_{R}\right) / 3 L \geqq \delta / 3 L .
$$

Finally, let either $x \in Y, y \notin Y$ or $x \notin Y, y \in Y$. We may assume that $x \in Q \in \mathscr{K}$ and $y \notin Y$. Then $\lambda_{Q} \leqq \delta$ and thus $2 / 3 L \leqq \Delta / \delta \leqq L+1 / 3 L$. Hence $f^{*}$ is $L^{*}$-bilipschitz. $\square$

3.3. Corollary. Let $n, q, L$, and $L^{*}$ be as in 3.2. Let $U \subset R^{n}$ be open, let $f: U \rightarrow R^{q}$ be an L-bilipschitz embedding, and let $\varepsilon \in C_{+}(U)$. Then there exists an $L^{*}$-bilipschitz PL embedding $g: U \rightarrow R^{q}$ such that $|g(x)-f(x)|<\varepsilon(x)$ for every $x \in U, \bar{g}|\partial U=\bar{f}| \partial U$, and, if $1 \leqq n=q \leqq 3, g U=f U$. Here $\bar{f}$ and $\bar{g}$ are the closed embeddings $\bar{U} \rightarrow R^{q}$ extending $f$ and $g$, respectively.

\section{CAT approximation of embeddings in manifolds}

4.1. Recall that CAT denotes either LQS or LIP. An immersion of a topological space into another is a continuous map which is locally an embedding. The following lemma for CAT $=$ LIP and $1 \leqq n=q \leqq 3$ is very similar to [37, Theorem 1].

4.2. Lemma. Let $(n, q)$ be admissible, let $(A, B)$ be either $\left(2 J^{n}, J^{n}\right)$ or $\left(2 J_{+}^{n}, J_{+}^{n}\right)$, let $f: A \rightarrow R^{q}$ be an embedding which is a CAT embedding on a neighborhood in $A$ of a closed subset $C$ of $A$, and let $\varepsilon \in C_{+}(B)$. Then there exists an embedding $f^{*}: A \rightarrow R^{q}$ such that 
(1) $f^{*}|A \backslash B=f| A \backslash B$,

(2) $f^{*} \mid B$ is PL,

(3) $f^{*} \mid U$ is a CAT embedding for some open neighborhood $U$ of $C$ in $A$,

(4) $\left|f^{*}(x)-f(x)\right|<\varepsilon(x)$ for every $x \in B$.

Consequently, $f^{*} \mid B \cup U$ is a CAT embedding.

Proof. We may assume that $C \subset(A \backslash B) \cap \bar{B}$. Then there exists a compact polyhedron $X$ which is a neighborhood of $C$ in $A$ and such that $f \mid X$ is a CAT embedding, $Y=X \cap B$ is the union of a subfamily of $\mathscr{L}_{n}$ locally finite in $Y$, and $Y$ is a PL manifold. Then also $Y_{1}=B \cap \overline{B \backslash Y}$ and $Y \cap Y_{1}$ are PL manifolds. Let $A_{1}=A \backslash I^{n}(3 / 2)$; then $\varepsilon_{1}=d\left(f B, f A_{1}\right) / 2>0$. Define $\varepsilon_{0} \in C_{+}(B)$ by

$$
\varepsilon_{0}(x)=\min \left(\varepsilon(x), \varepsilon_{1}, d(f(x), f(A \backslash B))\right) .
$$

By 1.9 (or also by 1.10 .2 if $n=q=2$ or 3 ) there exists $\delta \in C_{+}(Y)$ such that if $g \in U_{d}(f \mid Y, \delta)$ is a PL embedding, there is a PL embedding $g^{*} \in U_{d}\left(f \mid B, \varepsilon_{0}\right)$ extending g. Here $d(x, y)=|x-y|$.

Now $f \mid X$ is quasisymmetric if CAT=LQS or bilipschitz if CAT=LIP. Hence 2.20 and 3.2 imply that there is a CAT embedding $f_{1}: X \rightarrow R^{q}$ such that $f_{1}(x)=f(x)$ if $x \in X \backslash Y, f_{1} \mid Y$ is PL, and $\left|f_{1}(x)-f(x)\right|<\delta(x)$ if $x \in Y$.

Let $g^{*}$ be the extension of $g=f_{1} \mid Y$ given above. Define $f^{*}=(f \mid A \backslash B) \cup g^{*}$. Then $f^{*}$ is a continuous injection, and $f^{*}$ satisfies (1), .., (4) since $f^{*} \mid X=f_{1}$. Since $d\left(f^{*} B, f^{*} A_{1}\right) \geqq \varepsilon_{1}$, we conclude that $f^{*}$ is an embedding.

4.3. Remarks. 1. Suppose that $1 \leqq n=q \leqq 3$ and $(A, B)=\left(2 J^{n}, J^{n}\right)$ in 4.2. Then obviously $f^{*} B=f B$. Moreover, Alexander's trick [34, Proposition 3.22(i)] gives an isotopy $H$ rel $A \backslash B$ from $f$ to $f^{*}$ (i.e., an embedding $H: A \times I \rightarrow R^{q} \times I$ of the form $H(x, t)=\left(H_{t}(x), t\right)$ such that $H_{0}=f, H_{1}=f^{*}$, and $H_{t}(x)=f(x)$ if $(x, t) \in(A \backslash B) \times I)$; then $H_{t} B=f B$ for every $t \in I$.

2. It is easy to see that if $n=q=1$ and $(A, B)=\left(2 J_{+}^{1}, J_{+}^{1}\right)$ in 4.2 , one can choose $f^{*}$ in such a way that $f^{*} B=f B$.

4.4. Theorem. Let CAT $=\mathrm{LQS}$ or LIP, let $M$ and $N$ be CAT manifolds with $\partial N=\emptyset$ such that $M$ is n-dimensional and $N$ q-dimensional with either $n \leqq 1$, $q \geqq n$ or $n \geqq 2, q \geqq n+3$ or $2 \leqq n \leqq q \leqq 3$, let $U \subset M$ be an open neighborhood of $a$ closed set $C \subset M$, let $f: M \rightarrow N$ be an immersion such that $f \mid U$ is a CAT immersion, let $\varepsilon: M \backslash C \rightarrow(0, \infty)$ be continuous, and let $d$ be a metric defining the topology of $N$. Then there exists a CAT immersion $g: M \rightarrow N$ such that

(1) $g|C=f| C$,

(2) $d(g(x), f(x))<\varepsilon(x)$ for every $x \in M \backslash C$,

(3) $g M=f M$ if $n=q \leqq 3$ and $\partial M=\emptyset$,

(4) $g$ is a CAT embedding if $f$ is an embedding. 
Proof. In the case $n=0$ we can choose $g=f$ since $M$ is discrete. For the rest of the proof we assume that $n \geqq 1$, i.e., that $(n, q)$ is admissible. Let $\mathscr{A}$ and $\mathscr{B}$ be the CAT structures of $M$ and $N$, respectively. Replacing $\varepsilon$ by a smaller function we may assume that for every $x \in M \backslash C$ there is a chart $(Z, \psi) \in \mathscr{B}$ such that $\{y \in N \mid d(y, f(x))<\varepsilon(x)\} \subset Z$ and $\psi Z=R^{q}$.

Let $k=(n+1) M(n)$, where $M(n)=2^{n}$ is the number given in 2.9. We construct a locally finite family $\mathscr{V}$ of open relatively compact sets in $M$ and functions $r_{0}, r_{1}, \ldots, r_{k} \in C_{+}(M \backslash C)$ with the following properties:

(a) $f \mid \bar{V}$ is an embedding for every $V \in \mathscr{V}$.

(b) For each $V \in \mathscr{V}$ there is a chart $\left(V, \varphi_{V}\right) \in \mathscr{A}$ such that $\varphi_{V} V$ is either $2 J^{n}$ or $2 J_{+}^{n}$. We set $V^{*}=\varphi_{V}^{-1} J^{n}$ or $V^{*}=\varphi_{V}^{-1} J_{+}^{n}$, respectively; observe that $\bar{V}^{*} \subset V$. Let $\mathscr{V}^{*}=\left\{V^{*} \mid V \in \mathscr{V}\right\}$.

(c) $M \backslash U \subset \cup \mathscr{V}^{*} \subset \cup \mathscr{V} \subset M \backslash C$.

(d) $\mathscr{V}=\mathscr{V}_{1} \cup \ldots \cup \mathscr{V}_{k}$, where each $\mathscr{T}_{i}$ consists of disjoint sets.

(e) $r_{0}+\ldots+r_{k-1} \leqq r_{k}=\varepsilon$.

(f) $d(f V) \leqq \inf r_{0} V$ for every $V \in \mathscr{V}$.

(g) If $V \in \mathscr{V}$ and $i<k$, then $3 \sup r_{i} V \leqq \inf r_{i+1} V$.

To find $\mathscr{V}$ and the functions $r_{i}$, we choose first a closed neighborhood $C_{1} \subset U$ of $C$ in $M$. It is easy to find an open cover $\mathscr{U}$ of $M \backslash C$ consisting of chart neighborhoods relatively compact in $M \backslash C$ and functions $r_{0}, \ldots, r_{k} \in C_{+}(M \backslash C)$ such that (e) holds, (a), (f), and (g) hold if $\mathscr{V}$ is replaced by $\mathscr{U}$, and such that $V \cap C_{1}=\emptyset$ whenever $V \in \mathscr{U}$ and $V \leftarrow U$. By [31, Lemma 2.7] there is an open refinement $\mathscr{W}$ of $\mathscr{U}$ locally finite in $M \backslash C$ such that $\mathscr{W}=\mathscr{W}_{0} \cup \ldots \cup \mathscr{W}_{n}$ with the members of each $\mathscr{W}_{i}$ being disjoint. Then for each $i \leqq n$ there is a CAT homeomorphism $\varphi_{i}$ of $\cup \mathscr{W}_{i}$ onto an open subset $W_{i}$ of $R_{+}^{n}$. By 2.3 choose a cubical decomposition $\mathscr{K}_{i}$ of $W_{i}$ with respect to $W_{i}$, and let $\mathscr{K}_{i, 1}, \ldots, \mathscr{K}_{i, M(n)}$ be its partition as in 2.9. For $Q \in \mathscr{K}_{i}$ define $Q^{*} \subset W_{i}$ by $Q^{*}=\alpha_{Q} I^{n}(9 / 8)$ if $Q \cap R^{n-1}=\emptyset$ and by $Q^{*}=\alpha_{Q} I^{n}(9 / 8) \cap R_{+}^{n}$ if $Q \cap R^{n-1} \neq \emptyset$. Then $Q_{1}^{*} \cap Q_{2}^{*}=\emptyset$ if $Q_{1}, Q_{2} \in \mathscr{K}_{i}$ and $Q_{1} \cap Q_{2}=\emptyset$. For $Q \in \mathscr{K}_{i}$ choose a PL embedding $\sigma: Q^{*} \rightarrow R^{n}$ such that $\sigma Q^{*}=2 I^{n}$ and $\sigma Q=I^{n}(1 / 2)$ if $Q \cap R^{n-1}=\emptyset$ and such that $\sigma Q^{*}=2 I^{n} \cap R_{+}^{n}, \sigma\left(Q^{*} \cap R^{n-1}\right)=2 I^{n-1}$, and $\sigma Q=$ $I^{n}(1 / 2) \cap R_{+}^{n} \quad$ if $Q \cap R^{n-1} \neq 0$. Let $V_{Q}=\varphi_{i}^{-1} \sigma^{-1}\left(2 J^{n}\right)$ or $V_{Q}=\varphi_{i}^{-1} \sigma^{-1}\left(2 J_{+}^{n}\right)$, respectively, and let $\varphi_{V_{Q}}=\sigma \varphi_{i} \mid V_{Q}$. Then $\left(V_{Q}, \varphi_{V_{Q}}\right) \in \mathscr{A}$ satisfies (b). Let $\left\{F_{i} \mid 0 \leqq i \leqq n\right\}$ be a closed cover of $M \backslash C$ with $F_{i} \subset \cup \mathscr{W}_{i}$. Define

$$
\mathscr{V}_{i M(n)+j}=\left\{V_{Q} \mid Q \in \mathscr{K}_{i, j}, Q \cap \varphi_{i} F_{i} \neq \emptyset, V_{Q} \cap C_{1}=\emptyset\right\} .
$$

Then $\mathscr{V}=\mathscr{V}_{1} \cup \ldots \cup \mathscr{V}_{k}$ is the required family (observe that each $V \in \mathscr{V}$ is contained in some $W \in \mathscr{W})$, presented in such a way that (d) is satisfied.

By (c) there is a closed cover $\left\{C_{V} \mid V \in \mathscr{V}\right\} \cup\left\{C_{U}\right\}$ of $M$ such that $C_{V} \subset V^{*}$ for each $V \in \mathscr{V}$ and such that $C \subset C_{U} \subset U$. Let $\mathscr{V}_{0}=\{U\}$, and define a closed set $C^{i}=\cup\left\{C_{V} \mid V \in \mathscr{V}_{0} \cup \ldots \cup \mathscr{V}_{i}\right\}$ for $0 \leqq i \leqq k$.

We prove Theorem 4.4 by showing that there is a sequence $f=f_{0}, f_{1}, \ldots, f_{k}$ of continuous maps $M \rightarrow N$ such that 
(i) $f_{i}$ is a CAT immersion on a neighborhood of $C^{i}$ if $0 \leqq i \leqq k$,

(ii) $f_{i} \mid \bar{V}$ is an embedding if $V \in \mathscr{V}$ and $0 \leqq i \leqq k$,

(iii) $f_{i}$ is an embedding, $0 \leqq i \leqq k$, if $f$ is an embedding,

(iv) $d\left(f_{i} V\right) \leqq \inf r_{i} V$ if $V \in \mathscr{V}$ and $0 \leqq i \leqq k$,

(v) $d\left(f_{i}(x), f_{i-1}(x)\right)<r_{i-1}(x)$ if $x \in M \backslash C$ and $1 \leqq i \leqq k$,

(vi) $f_{i}(x)=f_{i-1}(x)$ if $x \in M \backslash \cup\left\{\bar{V}^{*} \mid V \in \mathscr{V}_{i}\right\}$ and $1 \leqq i \leqq k$.

We have already set $f_{0}=f$; this satisfies (i), ..., (iv). Assume that $1 \leqq m \leqq k$ and that we have defined $f_{i}$ for $0 \leqq i \leqq m-1$ satisfying (i), .., (vi). Let $V \in \mathscr{V}_{m}$ and $\varepsilon_{V}>0$; we specify $\varepsilon_{V}$ later. If $x, y \in V$, by (iv), (v), and (e), we have $d\left(f_{m-1}(y), f(x)\right)<r_{0}(x)+\ldots+r_{m-1}(x) \leqq \varepsilon(x)$. Hence there is a chart $(Z, \psi) \in \mathscr{B}$ such that $f_{m-1} V \subset Z$ and $\psi Z=R^{q}$. Set $A=\varphi_{V} V, B=\varphi_{V} V^{*}, h=\psi f_{m-1} \varphi_{V}^{-1}: A \rightarrow R^{q}$, and $X=\varphi_{V}\left(V \cap C^{m-1}\right)$. Then $h$ is an embedding by (ii), $X$ is closed in $A$, and $h$ is a CAT embedding on a neighborhood of $X$ in $A$ by (i). Let $\delta>0$. Then 4.2 gives an embedding $h^{*}: A \rightarrow R^{q}$ such that $h^{*}=h$ on $A \backslash B, h^{*} \mid B \cup E$ is a CAT embedding for some open neighborhood $E$ of $X$ in $A$, and $d\left(h^{*}, h\right)<\delta$. It follows that $f_{V}^{*}=$ $\psi^{-1} h^{*} \varphi_{V}: V \rightarrow N$ is an embedding which is a CAT embedding on a neighborhood of $V \cap C^{m}=\left(V \cap C^{m-1}\right) \cup C_{V}$ and coincides with $f_{m-1}$ on $V \backslash V^{*}$. We choose $\delta$ so small that $d\left(f_{V}^{*}(x), f_{m-1}(x)\right)<\varepsilon_{V}$ if $x \in V$. We now require that $\varepsilon_{V} \leqq \min r_{m-1} \bar{V}^{*}$. Since $\{W \in \mathscr{V} \mid \bar{W} \cap V \neq \emptyset\}$ is finite, we can also require, by (ii), that

$$
\varepsilon_{V} \leqq \frac{1}{3} d\left(f_{m-1}\left(\bar{W} \cap \bar{V}^{*}\right), f_{m-1}(\bar{W} \backslash V)\right) \quad \text { if } \quad W \in \mathscr{V} \text { and } \quad \bar{W} \cap V \neq \emptyset .
$$

By (iii) we can require the stronger condition

$$
\varepsilon_{V} \leqq \frac{1}{3} d\left(f_{m-1} \bar{V}^{*}, f_{m-1}(M \backslash V)\right)
$$

if $f$ is an embedding.

We choose a map $f_{V}^{*}: V \rightarrow N$ as above for each $V \in \mathscr{V}_{m}$. Then we can define a continuous map $f_{m}: M \rightarrow N$ by

$$
f_{m}(x)=\left\{\begin{array}{l}
f_{V}^{*}(x) \text { if } \quad x \in V \in \mathscr{V}_{m}, \\
f_{m-1}(x) \text { otherwise. }
\end{array}\right.
$$

Clearly $f_{m}$ satisfies (vi), (i), (v), and, by (g), also (iv). To prove (ii) and (iii), assume first that $f$ is an embedding. If $V \in \mathscr{V}_{m}, x \in \bar{V}^{*}$, and $y \in(M \backslash V) \backslash \cup\left\{\bar{W}^{*} \mid W \in \mathscr{V}_{m}\right\}$, then

$$
\begin{aligned}
d\left(f_{m}(x), f_{m}(y)\right) & \geqq d\left(f_{m-1}(x), f_{m-1}(y)\right)-\varepsilon_{V} \\
& \geqq \frac{2}{3} d\left(f_{m-1}(x), f_{m-1}(y)\right) .
\end{aligned}
$$

If $V, W \in \mathscr{V}_{m}, V \neq W, x \in \bar{V}^{*}$, and $y \in \bar{W}^{*}$, then

$$
\begin{aligned}
d\left(f_{m}(x), f_{m}(y)\right) & \geqq d\left(f_{m-1}(x), f_{m-1}(y)\right)-\varepsilon_{V}-\varepsilon_{W} \\
& \geqq \frac{1}{3} d\left(f_{m-1}(x), f_{m-1}(y)\right) .
\end{aligned}
$$


It follows that $f_{m}$ is an embedding. In exactly the same way one sees that $f_{m} \mid \bar{W}$ is an embedding for every $W \in \mathscr{V}$ if $f$ is only assumed to be an immersion. We have now constructed the sequence $f_{0}, \ldots, f_{k}$.

Consider $g=f_{k}$. By (i), $g$ is a CAT immersion of $C^{k}=M$ into $N$. Further, (vi) implies (1), (v) and (e) imply (2), and (iii) implies (4). Finally, suppose that $1 \leqq n=q \leqq 3$ and $\partial M=\emptyset$. Then in the above construction $f_{V}^{*} V=f_{m-1} V$ for each $V \in \mathscr{V}_{m}$, whence $f_{m} M=f_{m-1} M$. Thus $g M=f M$.

4.5. Remark. Theorem 4.4 holds for $n=q=1$ even if the supposition $\partial N=\emptyset$ is omitted, and we can choose $g$ in such a way that $g M=f M$ even if $\partial M \neq \emptyset$. By 4.3.2 this follows from the proof of 4.4 , the only modification being that one also allows the possibility $\psi Z=R_{+}^{1}$ in addition to $\psi Z=R^{1}$.

4.6. Corollary. Every CAT n-manifold, $n \geqq 0$, is CAT homeomorphic to a closed subset of $R^{2 n+1}$.

Proof. This follows from 1.15 and 4.4.

4.7. Remark. We give a stronger embedding result in 4.11. Corollary 4.6 implies that for every CAT manifold $(M, \mathscr{A})$ there is a metric $d$ on $M$ such that id: $(M, \mathscr{A}) \rightarrow(M, d)$ is a CAT homeomorphism, in which case $(M, d)$ is a metric CAT manifold. This shows that the two definitions of CAT manifolds are essentially equivalent. (To get full equivalence, we should identify two metric CAT manifolds $M_{i}=\left(M, d_{i}\right), i=1,2$, whenever id: $M_{1} \rightarrow M_{2}$ is a CAT homeomorphism.) It follows that the category of metric spaces and CAT immersions is a natural category in the sense of $[42,1.9]$.

4.8. Corollary. Every component of a CAT 1-manifold is CAT homeomorphic to exactly one of the following CAT 1-manifolds: $(0,1),[0,1),[0,1], \partial I^{2}$.

4.9. Corollary. Let $M$ and $N$ be homeomorphic CAT $n$-manifolds, $n \leqq 3$, and suppose that $\partial M=\emptyset=\partial N$ if $n=2$ or 3 . Then $M$ and $N$ are CAT homeomorphic.

4.10. Corollary. If $n=2$ or 3, every CAT $n$-manifold without boundary is CAT homeomorphic to a closed $C^{\infty}$-differentiable submanifold of $R^{2 n}$.

Proof. This follows from the proof of 1.17 and 4.9.

4.11. Corollary. Every CAT n-manifold, $n \geqq 1$, can be closedly CAT embedded into $R^{2 n}$.

Proof. The case $n=1$ follows from 4.8 and the case $n=2$ or 3 from 1.4 and 4.10. If $n \geqq 4$ (or, in fact, if $n \neq 2$ ), then 4.11 follows from 1.17 and 4.4.

4.12. Corollary. If $M$ is a LIP $n$-manifold, $n \geqq 1$, there is a closed locally LIP flat LIP embedding $f: M \rightarrow R^{3 n}$. 
Proof. This follows from 4.11 and [26, Corollary 4.8].

4.13. Corollary. Let $n \geqq 0, q \geqq 2 n+1$, and let $M$ be a LIP $n$-manifold, $N$ a LIP $q$-manifold, $f: M \rightarrow N$ continuous, $C \subset M$ closed, $U$ a closed neighborhood of $C, f \mid U$ a closed LIP embedding, $f C \subset$ int $N$, and $\mathscr{U}$ an open cover of $N$. Then $N(f, \mathscr{U})$ contains a LIP embedding $g: M \rightarrow \operatorname{int} N$ with $g|C=f| C$.

This also holds if LIP is replaced by LQS and $C=\emptyset$.

Proof. We may assume that $f M \subset$ int $N$ by [25, Lemma 2.3 and 1.2]. By 1.13, $f \mid U$ is a $Z^{n}$-embedding. Hence we may assume by 1.12 that $f$ is an embedding. The corollary now follows from 4.4 .

4.14. Remarks. 1. Consider the case CAT=LIP. The special case of 4.4 in which $n=q \leqq 3$ and $f$ is a homeomorphism is [37, Theorem 2]. There the result is stated for manifolds with boundary, but the proof is only valid for manifolds without boundary. However, the first-named author could reduce (using 4.14.6) the case with possibly $\partial M \neq \emptyset$ to the case $\partial M=\emptyset$. Another proof will be mentioned in 4.14.3. Corollary 4.6 solves affirmatively [26, Problem 9.1(1)] and with 4.11 improves [26, Theorems 4.2 and 4.5], which only give a closed LIP embedding of a LIP $n$-manifold into $R^{n(n+1)}(n \geqq 1)$. Similarly 4.12 improves [26, Theorem 4.9]. In [41, Theorem 3.8], 4.9 is proved for 2-manifolds with boundary. In fact, [41, Theorem 3.7] and PL approximation results imply a (stronger) special case of 4.4 for these manifolds.

2. Theorem 4.4 for $\mathrm{CAT}=\mathrm{LQS}, n=q \leqq 3$, and $f$ a homeomorphism was conjectured in $[37, \S 4$, p. 138]. Corollary 4.6 for $\mathrm{CAT}=\mathrm{LQS}$ solves a problem in $[44,4.3]$. The result that every compact metric LQS manifold can be quasisymmetrically embedded into a Euclidean space also follows from [1, Remarque 2, p. 732, and Proposition 2 (h), (i)] by [38, Theorem 2.10] (cf. also [38, Remark 3.20]). Kuusalo [21] and Cannon [9] have considered orientable quasiconformal 2-manifolds. They did not assume that these are metrizable, but they supposed there to exist a locally quasiconformal atlas the dilatations of whose coordinate changes have certain boundedness properties. They proved that these manifolds have a locally quasiconformally equivalent conformal structure and, hence, are metrizable. In particular, a corollary of [21, Satz 3] is that, in our terminology, every orientable connected LQS 2-manifold without boundary is LQS homeomorphic to a Riemann surface; cf. 4.10. The metrizability result is generalized for $n \geqq 2$ in [22].

3. Tukia and Väisälä [39, Theorems 4.4 and 4.8] have recently proved, using Sullivan's methods [36], that if $M, N$ are CAT manifolds with $\operatorname{dim} M \neq 4 \neq \operatorname{dim} \partial M$, every homeomorphism of $M$ onto $N$ can be relatively approximated by CAT homeomorphisms. Sullivan proved this in [36, Corollary 3] for LIP manifolds without boundary. In [14, Theorem 3.11] one proves that every CAT manifold satisfying the above dimension condition and homeomorphic either to $R^{n}, S^{n}$, or $I^{n}$ is CAT homeomorphic to it. Earlier this was proved for CAT=LIP in [26, Section 8]. 
4. We can omit the hypothesis $\partial N=\emptyset$ (but still have $g M \subset$ int $N$ ) in the weaker form of 4.4 where $C=\emptyset$ and (3) is omitted, provided that the map $M \rightarrow f M$ defined by $f$ is proper (which is satisfied if $f$ is an embedding). To see this, observe first that since $f M$ is locally compact, we may assume that $f M$ is closed in $N$, in which case by [19, Lemma, p. 47] there is $\delta \in C_{+}(N)$ with $\delta(f(x)) \leqq \varepsilon(x) / 2$ if $x \in M$. Then, using a collar of $\partial N$ in $N$, we can construct an embedding $h: N \rightarrow N$ in $U_{d}\left(\mathrm{id}_{N}, \delta\right)$ with $h N \subset$ int $N([25$, Lemma 2.3]). Hence 4.4 gives a CAT immersion $g: M \rightarrow N$ such that $g M \subset$ int $N, 4.4(4)$ holds, and $g \in U_{d}(h f, \varepsilon / 2)$, whence $g \in U_{d}(f, \varepsilon)$.

5. Let $(n, q)$ be admissible, $M$ a CAT $n$-manifold, $N$ a PL $q$-manifold, $f: M \rightarrow N$ an embedding, $\varepsilon \in C_{+}(M)$, and $d$ a metric for $N$ such that $\mathrm{jd}: N \rightarrow(N, d)$ is a LIP homeomorphism. Then $U_{d}(f, \varepsilon)$ contains a CAT embedding $g: M \rightarrow N$ such that there is a closed subset $Y$ of $g M$ whose $n$-dimensional Hausdorff measure is zero if $\mathrm{CAT}=\mathrm{LQS}$ or whose Hausdorff dimension is $\leqq n-1$ if $\mathrm{CAT}=\mathrm{LIP}$ and for which $g M \backslash Y$ is a PL submanifold of $N$. In fact, since we may assume that $\partial N=\emptyset$ by 4.14.4, the proof of 4.4 gives the required $g$ if one chooses $U=\emptyset$ and each $(Z, \psi)$ such that $\psi$ is PL. To see this for CAT $=\mathrm{LQS}$, one can use the fact $([43$, Theorem 4.1]) that every quasisymmetric image in $R^{n}$ of an open set in $R^{p}, p<n$, has zero Lebesgue measure.

6. Suppose that $1 \leqq n=q \leqq 3, \partial M=\emptyset$, and that $f$ is an embedding in 4.4. Then the construction of $g$ allows one also to construct, by 4.3.1, an isotopy $H$ rel $C$ from $f$ to $g$ such that $H_{t} M=f M$ if $t \in I$ and $d\left(H_{t}(x), f(x)\right) \leqq \varepsilon(x)$ if $(x, t) \in(M \backslash C) \times I$.

\section{References}

[1] Assouad, P.: Étude d'une dimension métrique liée à la possibilité de plongements dans $R^{n}$. C. R. Acad. Sci. Paris Sér. A-B 288, 1979, A731-A734.

[2] Bing, R. H.: Locally tame sets are tame. - Ann. of Math. (2) 59, 1954, 145-158.

[3] BING, R. H.: Approximating surfaces with polyhedral ones. - Ibid. 65, 1957, 456-483.

[4] BING, R. H.: An alternative proof that 3-manifolds can be triangulated. - Ibid. 69, 1959, $37-65$.

[5] Brown, E. M.: The Hauptvermutung for 3-complexes. - Trans. Amer. Math. Soc. 144, 1969, 173-196.

[6] BRyant, J. L.: Approximating embeddings of polyhedra in codimension three. - Ibid. 170, 1972, 85-95.

[7] Bryant, J. L., and C. L. Seebeck III: Locally nice embeddings in codimension three. - Quart. J. Math. Oxford Ser. (2) 21, 1970, 265-272.

[8] CaIRns, S. S.: Homeomorphisms between topological manifolds and analytic manifolds. Ann. of Math. (2) 41, 1940, 796-808.

[9] CANnon, R. J., JR.: Quasiconformal structures and the metrization of 2-manifolds. - Trans. Amer. Math. Soc. 135, 1969, 95-103.

[10] Carleson, L.: The extension problem for quasiconformal mappings. - Contributions to analysis, edited by L. V. Ahlfors et al., Academic Press, New York-London, 1974, 39-47. 
[11] CRAGGS, R.: Small ambient isotopies of a 3-manifold which transform one embedding of a polyhedron into another. - Fund. Math. 68, 1970, 225-256.

[12] Dancis, J.: General position maps for topological manifolds. - Geometric topology (Proc. Georgia Topology Conf., Athens, Ga., 1977), edited by J. C. Cantrell, Academic Press, New'York-San Francisco--London, 1979, 305-321.

[13] Edwards, R. D.: The equivalence of close piecewise linear embeddings. - General Topology Appl. 5, 1975, 147-180.

[14] GAULD, D. B., and J. VÄISÄLÄ: Lipschitz and quasiconformal flattening of spheres and cells. Ann. Acad. Sci. Fenn. Ser. A I Math. 4, 1978/79, 371-382.

[15] Hirsch, M. W.: On imbedding differentiable manifolds in euclidean space. - Ann. of Math. (2) 73, 1961, 566-571.

[16] Hudson, J. F. P.: Piecewise linear topology. - W. A. Benjamin, Inc., New York-Amsterdam, 1969.

[17] Hurewicz, W., and H. Wallman: Dimension theory. - Princeton University Press, Princeton, 1948.

[18] KIIKKA, M.: Piecewise linear approximation of quasiconformal and Lipschitz homeomorphisms. - Ann. Acad. Sci. Fenn. Ser. A I Math. Dissertationes 30, 1980, 1-24.

[19] Kirby, R. C., and L. C. Siebenmann: Foundational essays on topological manifolds, smoothings, and triangulations. - Annals of Mathematics Studies 88, Princeton University Press and University of Tokyo Press, Princeton, N. J., 1977.

[20] Kobayashi, S., and K. Nomizu: Foundations of differential geometry, Vol. I. - Interscience Publishers, New York-London, 1963.

[21] Kuusalo, T.: Verallgemeinerter Riemannscher Abbildungssatz und quasikonforme Mannigfaltigkeiten. - Ann. Acad. Sci. Fenn. Ser. A I Math. 409, 1967, 1-24.

[22] KuUsalo, T.: Generalized conformal capacity and quasiconformal metrics. - Proceedings of the Romanian-Finnish seminar on Teichmüller spaces and quasiconformal mappings, Braşov, 1969, edited by Cabiria Andreian Cazacu, Publishing House of the Academy of the Socialist Republic of Romania, Bucharest, 1971, 193-202.

[23] LeEs, J. A.: Locally flat imbeddings of topological manifolds. - Ann. of Math. (2) 89, 1969, $1-13$.

[24] Lehto, O., and K. I. Virtanen: Quasiconformal mappings in the plane. - Springer-Verlag, Berlin-Heidelberg-New York, 1973.

[25] LuUkKainen, J.: Approximating continuous maps of metric spaces into manifolds by embeddings. - Math. Scand. 49, 1981 (to appear).

[26] LuUkKainen, J., and J. VäISÄLÄ: Elements of Lipschitz topology. - Ann. Acad. Sci. Fenn. Ser. A I Math. 3, 1977, 85-122.

[27] Matsumoto, Y.: Wild embeddings of piecewise linear manifolds in codimension two. - Geometric topology (Proc. Georgia Topology Conf., Athens, Ga., 1977), edited by J. C. Cantrell, Academic Press, New York-San Francisco-London, 1979, 393428.

[28] Miller, R. T.: Approximating codimension 3 embeddings. - Ann. of Math. (2) 95, 1972, 406- 416.

[29] Morse, E. E.: Affine structures in 3-manifolds, V. The triangulation theorem and Hauptvermutung. - Ibid. 56, 1952, 96-114.

[30] MoIsE, E. E.: Geometric topology in dimensions 2 and 3. - Springer-Verlag, New YorkHeidelberg-Berlin, 1977.

[31] Munkres, J. R.: Elementary differential topology. - Annals of Mathematics Studies, No. 54, Princeton University Press, Princeton, N. J., 1963.

[32] Pedersen, E. K.: Embeddings of topological manifolds. - Illinois J. Math. 19, 1975, 440 - 447. 
[33] RengGli, H.: Doppelverhältnisse und quasikonforme Abbildungen. - Comment. Math. Helv. $43,1968,161-175$.

[34] Rourke, C. P., and B. J. SANDERSON: Introduction to piecewise-linear topology. - SpringerVerlag, Berlin-Heidelberg-New York, 1972.

[35] Rushing, T. B.: Topological embeddings. - Academic Press, New York-London, 1973.

[36] Sullivan, D.: Hyperbolic geometry and homeomorphisms. - Geometric topology (Proc. Georgia Topology Conf., Athens, Ga., 1977), edited by J. C. Cantrell, Academic Press, New York-San Francisco-London, 1979, 543-555.

[37] TukiA, P.: Lipschitz approximation of homeomorphisms. - Ann. Acad. Sci. Fenn. Ser. A I Math. 4, 1978/1979, 137-144.

[38] TukIA, P., and J. VÄISÄLÄ: Quasisymmetric embeddings of metric spaces. - Ibid. 5, 1980, 97-114.

[39] TukIA, P., and J. VÄISÄLÄ: Lipschitz and quasiconformal approximation and extension. - Ibid. 6, 1981, 303-342.

[40] VÄISÄLÄ, J.: Lectures on $n$-dimensional quasiconformal mappings. - Lecture Notes in Mathematics 229, Springer-Verlag, Berlin-Heidelberg-New York, 1971.

[41] V̈̈ıÄ̈̈̈̈, J.: Piecewise linear approximation of lipeomorphisms. - Ann. Acad. Sci. Fenn. Ser. A I Math. 3, 1977, 377-383.

[42] 'V̈̈ıs̈̈L̈̈, J.: An axiomatic approach to the theory of manifolds. - Rev. Roumaine Math. Pures Appl. 24, 1979, 489-503.

[43] VÄISÄLÄ, J.: Quasi-symmetric embeddings in Euclidean spaces. - Trans. Amer. Math. Soc. 264, 1981, 191-204.

[44] Väıs̈̈L̈̈, J.: Lipschitz topology. - International topological conference (Moscow, 1979) (to appear).

[45] Venema, G. A.: Approximating disks in 4-space. - Michigan Math. J. 25, 1978, 19-27.

[46] Whitney, H.: The self-intersections of a smooth $n$-manifold in $2 n$-space. - Ann. of Math. (2) $45,1944,220-246$.

University of Helsinki

Department of Mathematics

SF-00100 Helsinki 10

Finland

Received 8 May 1981 